\title{
Pacific
}

Journal of

Mathematics

\section{PREALTERNATIVE ALGEBRAS \\ AND PREALTERNATIVE BIALGEBRAS}

Xiang Ni And Chengming BaI 


\title{
PREALTERNATIVE ALGEBRAS AND PREALTERNATIVE BIALGEBRAS
}

\author{
XiAng Ni And Chengming BAi
}

\begin{abstract}
We introduce a notion of prealternative algebra, which may be viewed as an alternative algebra whose product can be decomposed into two compatible pieces. It is also an alternative algebra analogue of a dendriform dialgebra or a pre-Lie algebra. The left and right multiplication operators of a prealternative algebra give a bimodule structure of the associated alternative algebra. There exists a (coboundary) bialgebra theory for prealternative algebras, namely, prealternative bialgebras, which exhibits all the familiar properties of the Lie bialgebra theory. In particular, a prealternative bialgebra is equivalent to a phase space of an alternative algebra, and our study leads to what we call the PA equations in a prealternative algebra, which are analogues of the classical Yang-Baxter equation.
\end{abstract}

\section{Introduction}

A dendriform dialgebra is a vector space $D$ together with two bilinear operations $\prec, \succ: D \otimes D \rightarrow D$ such that for any $x, y, z \in D$

$$
\begin{aligned}
& (x \prec y) \prec z=x \prec(y \circ z), \\
& (x \succ y) \prec z=x \succ(y \prec z), \\
& (x \circ y) \succ z=x \succ(y \succ z),
\end{aligned}
$$

where $x \circ y=x \prec y+x \succ y$. Dendriform dialgebras were introduced by J.-L. Loday in 1995 as the (Koszul) dual of the associative dialgebra, which is related to periodicity phenomena in algebraic K-theory [Loday 2001]. It was further studied in connection with several areas in mathematics and physics, including operads [Loday 2004], homology [Frabetti 1997; 1998], Hopf algebras [Chapoton 2002; Holtkamp 2004; Ronco 2002], Lie and Leibniz algebras [Frabetti 1998], combinatorics [Aguiar and Sottile 2005; 2006], arithmetic [Loday 2002] and quantum

MSC2000: 17D05, 17A30, 16W30.

Keywords: alternative algebra, prealternative algebra, prealternative bialgebra, classical

Yang-Baxter equation.

This work was supported in part by NSFC (10621101, 10920161), NKBRPC (2006CB805905) and SRFDP (200800550015). 
field theory [Ebrahimi-Fard and Guo 2007]. See [Loday 2001] for a beautiful introduction and motivation of this subject.

For any dendriform dialgebra $(D, \prec, \succ)$, the bilinear operation $\circ$ defines an associative algebra. Thus, a dendriform dialgebra may be seen as an associative algebra whose multiplication can be decomposed into two coherent operations.

We may reexamine the identity (1-1) as follows. Let $A$ be a vector space together with two operations $\prec, \succ: A \otimes A \rightarrow A$. The right associator (r-associator), middle associator (m-associator) and left associator (l-associator) are defined for all $x, y, z \in A$ by

$$
\begin{aligned}
(x, y, z)_{r} & =(x \prec y) \prec z-x \prec(y \circ z), \\
(x, y, z)_{m} & =(x \succ y) \prec z-x \succ(y \prec z), \\
(x, y, z)_{l} & =(x \circ y) \succ z-x \succ(y \succ z),
\end{aligned}
$$

respectively, where $x \circ y=x \prec y+x \succ y$. So $(D, \prec, \succ)$ is a dendriform dialgebra if and only if all the above three associators are zero.

On the other hand, alternative algebras are a class of important nonassociative algebras [Kuz'min and Shestakov 1995; Schafer 1952; 1954]. Alternative algebras are closely related to Lie algebras [Schafer 1954], Jordan algebras [Jacobson 1968] and Malcev algebras [Kuz'min and Shestakov 1995]. Due to the relationships between associative algebras and alternative algebras (see Section 2), it is natural to consider the algebraic structure on an alternative algebra as an analogue of a dendriform dialgebra on an associative algebra. So we introduce a notion of prealternative algebra, one of the main objects in this paper. Just as an alternative algebra is a generalization of an associative algebra that weakens the condition of associativity, a prealternative algebra is a generalization of a dendriform dialgebra that weakens the conditions of $l$-associativity, $m$-associativity and $r$-associativity.

There has already been a Lie algebraic version of the relationship between associative algebras and dendriform dialgebras. A class of nonassociative algebras, the pre-Lie algebras (also called left-symmetric algebras, Vinberg algebras and so on - see a survey article [Burde 2006] and the references therein) play a role similar to dendriform dialgebras. Therefore, in this sense, prealternative algebras are just alternative algebra analogues of pre-Lie algebras or dendriform dialgebras.

Goncharov [2007] constructed alternative D-bialgebras, a bialgebra theory for alternative algebras. In this paper, we show that prealternative bialgebras serve as a (coboundary) bialgebra theory for prealternative algebras, and exhibit all the familiar properties of the Lie bialgebra theory of Drinfeld [1983]. Just as an alternative D-bialgebra is equivalent to an alternative analogue of Manin triple [Goncharov 2007; Chari and Pressley 1994], a prealternative bialgebra is equivalent to a phase space of an alternative algebra [Kupershmidt 1994; Bai 2006]. In particular, there 
exists an unexpected Drinfeld double construction for a prealternative bialgebra. Also, there is a clear analogy between alternative D-bialgebras and prealternative bialgebras. On the other hand, we emphasize that the representation theories of alternative and prealternative algebras play an essential role in establishing the bialgebra theories. We also point out that both alternative D-bialgebras and prealternative bialgebras can be fit into the general framework of generalized bialgebras introduced in [Loday 2008]. So it would be interesting to find the relationship to Loday's question, that is, to find, as he put it, "good triples of operads".

The paper is organized as follows. In Section 2, we study bimodules of alternative algebras and introduce various methods to construct prealternative algebras. In Section 3, we recall the properties of alternative D-bialgebras of Goncharov and prove some new results. In Section 4, we generalize the notion of phase space in mathematical physics [Kupershmidt 1994] to the realm of alternative algebras, and show that prealternative algebras are the natural underlying structures. In Section 5, we define and study bimodules and matched pairs of prealternative algebras. In Section 6, we introduce the notion of prealternative bialgebra, which is equivalent to a phase space of an alternative algebra. In Section 7, we show that there is a reasonable coboundary (prealternative) bialgebra theory; what we study leads to what we call PA equations. Section 8 discusses the properties of the PA equations. We compare alternative D-bialgebras and prealternative algebras in Section 9. In the appendix, we prove the main results in [Goncharov 2007] by a somewhat different approach; we point out a Drinfeld double construction for an alternative $D$-bialgebra that was not given there.

Throughout this paper, all the algebras are finite-dimensional over a fixed base field $\mathbf{k}$ of characteristic not 2 . We give some notations as follows.

Let $V$ be a vector space. Let $\mathfrak{B}: V \otimes V \rightarrow \mathbb{F}$ be a symmetric or skew-symmetric bilinear form on $V$. If $W$ is a subspace of $V$, then we define

$$
W^{\perp}=\{x \in V \mid \mathfrak{B}(x, y)=0 \text { for all } y \in W\} .
$$

We say $W$ is isotropic if $W \subset W^{\perp}$ and Lagrangian if $W=W^{\perp}$.

Let $(A, \diamond)$ be a vector space with a binary operation $\diamond: A \otimes A \rightarrow A$. Let $\mathfrak{l}_{\diamond}(x)$ and $\mathfrak{r}_{\diamond}(x)$ denote the left and right multiplication operators, that is, $\mathfrak{l}_{\diamond}(x) y=\mathfrak{r}_{\diamond}(y) x=$ $x \diamond y$ for any $x, y \in A$. We may sometimes write instead $\mathfrak{l}(x)$ or $\mathfrak{r}(x)$ when no confusion would result. Let $\mathfrak{l}_{\diamond}, \mathfrak{r}_{\diamond}: A \rightarrow \mathfrak{g l}(A)$ be two linear maps with $x \mapsto \mathfrak{l}_{\diamond}(x)$ and $x \mapsto \mathfrak{r}_{\diamond}(x)$, respectively.

Let $V$ be a vector space and $r=\sum_{i} a_{i} \otimes b_{i} \in V \otimes V$. Set

$$
r_{12}=\sum_{i} a_{i} \otimes b_{i} \otimes 1, \quad r_{13}=\sum_{i} a_{i} \otimes 1 \otimes b_{i}, \quad r_{23}=\sum_{i} 1 \otimes a_{i} \otimes b_{i},
$$


where 1 is a symbol playing a role similar to the unit. If in addition there exists a binary operation $\diamond: V \otimes V \rightarrow V$ on $V$, then the operation between two of the $r$ is done in the obvious way. For example,

$$
\begin{aligned}
& r_{12} \diamond r_{13}=\sum_{i, j} a_{i} \diamond a_{j} \otimes b_{i} \otimes b_{j}, \quad r_{13} \diamond r_{23}=\sum_{i, j} a_{i} \otimes a_{j} \otimes b_{i} \diamond b_{j}, \\
& r_{23} \diamond r_{12}=\sum_{i, j} a_{j} \otimes a_{i} \diamond b_{j} \otimes b_{i} .
\end{aligned}
$$

Let $V$ be a vector space. Let $\sigma: V \otimes V \rightarrow V \otimes V$ be the flip defined by

$$
\sigma(x \otimes y)=y \otimes x \quad \text { for all } x, y \in V .
$$

We call $r \in V \otimes V$ symmetric if $r=\sigma(r)$ and skew-symmetric if $r=-\sigma(r)$. On the other hand, any $r \in V \otimes V$ can be identified as a linear map $T_{r}: V^{*} \rightarrow V$ via

$$
\left\langle u^{*} \otimes v^{*}, r\right\rangle=\left\langle u^{*}, T_{r}\left(v^{*}\right)\right\rangle \quad \text { for all } u^{*}, v^{*} \in V^{*},
$$

where $\langle\cdot, \cdot\rangle$ is the canonical paring between $V$ and $V^{*}$. We call $r \in V \otimes V$ nondegenerate if $T_{r}$ is invertible. Any invertible linear map $T: V^{*} \rightarrow V$ induces a nondegenerate bilinear form $\mathfrak{B}$ on $V$ by

$$
\mathfrak{B}(u, v)=\left\langle T^{-1} u, v\right\rangle \quad \text { for all } u, v \in V .
$$

We call $T$ symmetric (respectively skew-symmetric) if the induced bilinear form $\mathfrak{B}$ is symmetric (respectively skew-symmetric). Obviously, the symmetry or skewsymmetry of both $T$ and the corresponding $r \in V \otimes V$ coincide.

Let $V_{1}, V_{2}$ be two vector spaces and $T: V_{1} \rightarrow V_{2}$ be a linear map. Denote the dual (linear) map by $T^{*}: V_{2}^{*} \rightarrow V_{1}^{*}$ defined by

$$
\left\langle v_{1}, T^{*}\left(v_{2}^{*}\right)\right\rangle=\left\langle T\left(v_{1}\right), v_{2}^{*}\right\rangle \quad \text { for all } v_{1} \in V_{1}, v_{2}^{*} \in V_{2}^{*} .
$$

On the other hand, $T$ can be identified as an element $r_{T} \in V_{2} \otimes V_{1}^{*}$ by

$$
\left\langle r_{T}, v_{2}^{*} \otimes v_{1}\right\rangle=\left\langle T\left(v_{1}\right), v_{2}^{*}\right\rangle \quad \text { for all } v_{1} \in V_{1}, v_{2}^{*} \in V_{2}^{*} .
$$

Note that (1-7) is exactly the case that $V_{1}=V_{2}^{*}$. In the above sense, any linear map $T: V_{1} \rightarrow V_{2}$ is obviously an element in $\left(V_{2} \oplus V_{1}^{*}\right) \otimes\left(V_{2} \oplus V_{1}^{*}\right)$.

Let $A$ be an algebra and $V$ be a vector space. For any linear map $\rho: A \rightarrow \mathfrak{g l}(V)$, define a linear map $\rho^{*}: A \rightarrow \mathfrak{g l}\left(V^{*}\right)$ by

$$
\left\langle\rho^{*}(x) v^{*}, u\right\rangle=\left\langle v^{*}, \rho(x) u\right\rangle \quad \text { for all } x \in A, u \in V, v^{*} \in V^{*} .
$$

Note that in this case $\rho^{*}$ not the map dual to $\rho$ in the sense of (1-9).

For vector spaces $V_{1}$ and $V_{2}$, we denote the elements of $V_{1} \oplus V_{2}$ by $u+v$ or $(u, v)$ for $u \in V_{1}$ and $v \in V_{2}$.

We may use 1 to denote the identity transformation of a vector space $V$. 


\section{Representation theory of alternative algebras and prealternative algebras}

Definition 2.1. An alternative algebra $(A, \circ)$ is a vector space $A$ equipped with a bilinear operation $(x, y) \rightarrow x \circ y$ satisfying

$$
(x, x, y)=(y, x, x)=0 \quad \text { for all } x, y, z \in A,
$$

where $(x, y, z)=(x \circ y) \circ z-x \circ(y \circ z)$ is the associator.

Remark 2.2. If the characteristic of the field is not 2, then an alternative algebra $(A, \circ)$ also satisfies for all $x_{1}, x_{2}, y \in A$ the stronger axioms

$$
\left(x_{1}, x_{2}, y\right)+\left(x_{2}, x_{1}, y\right)=0 \text { and }\left(y, x_{1}, x_{2}\right)+\left(y, x_{2}, x_{1}\right)=0 .
$$

Definition 2.3 [Schafer 1952]. Let $(A, \circ)$ be an alternative algebra and $V$ be a vector space. Let $L, R: A \rightarrow \mathfrak{g l}(V)$ be two linear maps. We call $V$ (or the pair $(L, R)$, or $(V, L, R))$ a representation or a bimodule of $A$ if for any $x, y \in A$

$$
L\left(x^{2}\right)=L(x) L(x), \quad R\left(x^{2}\right)=R(x) R(x)
$$

and

$$
\begin{aligned}
R(y) L(x)-L(x) R(y) & =R(x \circ y)-R(y) R(x), \\
L(y \circ x)-L(y) L(x) & =L(y) R(x)-R(x) L(y) .
\end{aligned}
$$

By [Schafer 1995], $(V, L, R)$ is a bimodule of an alternative algebra $(A, \circ)$ if and only if the direct sum $A \oplus V$ of vector spaces is turned into an alternative algebra (the semidirect sum) by defining multiplication in $A \oplus V$ by

$$
\left(x_{1}+v_{1}\right) *\left(x_{2}+v_{2}\right)=x_{1} \circ x_{2}+\left(L\left(x_{1}\right) v_{2}+R\left(x_{2}\right) v_{1}\right)
$$

for all $x_{1}, x_{2} \in A$ and $v_{1}, v_{2} \in V$.

We denote it by $A \ltimes_{L, R} V$ or simply $A \ltimes V$.

Proposition 2.4. If $(V, L, R)$ is a bimodule of an alternative algebra $(A, \circ)$, then $\left(V^{*}, R^{*}, L^{*}\right)$ is a bimodule of $(A, \circ)$.

Proof. By (2-2) and (2-3), we have

$$
L(x \circ y)-L(x) L(y)=-L(y \circ x)+L(y) L(x)=R(x) L(y)-L(y) R(x)
$$

for all $x, y \in A$. So for any $u^{*} \in V^{*}, v \in V$, we have

$$
\begin{aligned}
\left\langle\left(L^{*}(y) R^{*}(x)-R^{*}(x) L^{*}(y)\right) u^{*}, v\right\rangle & =\left\langle u^{*},(R(x) L(y)-L(y) R(x)) v\right\rangle \\
& =\left\langle u^{*},(L(x \circ y)-L(x) L(y)) v\right\rangle \\
& =\left\langle\left(L^{*}(x \circ y)-L^{*}(y) L^{*}(x)\right) u^{*}, v\right\rangle .
\end{aligned}
$$

So $L^{*}(y) R^{*}(x)-R^{*}(x) L^{*}(y)=L^{*}(x \circ y)-L^{*}(y) L^{*}(x)$. Similarly, $\left(V^{*}, R^{*}, L^{*}\right)$ also satisfies the other axioms defining a bimodule of $(A, \circ)$. 
Definition 2.5. A prealternative algebra $(A, \prec, \succ)$ is a vector space $A$ with two bilinear operations denoted by $\prec, \succ: A \otimes A \rightarrow A$ satisfying

$$
\begin{aligned}
(x, y, z)_{m}+(y, x, z)_{r} & =0, \\
(x, y, z)_{m}+(x, z, y)_{l} & =0, \quad(y, x, x)_{r}=(x, x, y)_{l}=0
\end{aligned}
$$

for all $x, y, z \in A$, where $(x, y, z)_{r},(x, y, z)_{m},(x, y, z)_{l}$ are defined by (1-2) and $x \circ y=x \succ y+x \prec y$.

Remark 2.6. If the characteristic of the field is not 2, then a prealternative algebra $(A, \prec, \succ)$ satisfies for any $x, y, z \in A$ the strong axioms

$$
\begin{aligned}
(x, y, z)_{m}+(y, x, z)_{r} & =0, & (x, y, z)_{m}+(x, z, y)_{l} & =0, \\
(x, y, z)_{l}+(y, x, z)_{l} & =0, & (x, y, z)_{r}+(x, z, y)_{r} & =0 .
\end{aligned}
$$

It would be interesting to describe free prealternative algebras; see [Loday 2001]. Proposition 2.7. Let $(A, \prec, \succ)$ be a prealternative algebra. Then the operation

$$
x \circ y=x \succ y+x \prec y \quad \text { for all } x, y \in A,
$$

defines an alternative algebra, which is called the associated alternative algebra of $A$ and denoted by $\operatorname{Alt}(A)$. We call $(A, \prec, \succ)$ a compatible prealternative algebra structure on the alternative algebra $\operatorname{Alt}(A)$.

Proof. In fact, for any $x, y \in A$, we have

$$
\begin{aligned}
(x, x, y)= & (x \circ x) \circ y-x \circ(x \circ y) \\
= & (x \circ x) \succ y+(x \succ x) \prec y+(x \prec x) \prec y \\
& \quad-x \succ(x \succ y)-x \succ(x \prec y)-x \prec(x \circ y) \\
= & (x, x, y)_{l}+(x, x, y)_{m}+(x, x, y)_{r}=0 .
\end{aligned}
$$

Similarly, we show that $(y, x, x)=0$.

Remark 2.8. Thus a prealternative algebra can be viewed as an alternative algebra whose operation decomposes into two compatible pieces. On the other hand, it is obvious that an associative algebra is an alternative algebra and a dendriform dialgebra is a prealternative algebra.

If $(A, \circ)$ is an alternative algebra, then $\left(A, \mathfrak{l}_{\circ}, \mathfrak{r}_{\circ}\right)$ is a bimodule of $A$.

Proposition 2.9. Let $(A, \prec, \succ)$ be a prealternative algebra. Then $\left(A, \mathfrak{l}_{\succ}, \mathfrak{r}_{\prec}\right)$ is a bimodule of the associated alternative algebra $(\operatorname{Alt}(A), \circ)$.

Proof. For any $x, y, z \in A$, we have

$$
\begin{aligned}
\left(\mathfrak{r}_{\prec}(y) \mathfrak{l}_{\succ}(x)-\mathfrak{l}_{\succ}(x) \mathfrak{r}_{\prec}(y)\right) z & =(x \succ z) \prec y-x \succ(z \prec y)=z \prec(x \circ y)-(z \prec x) \prec y \\
& =\left(\mathfrak{r}_{\prec}(x \circ y)-\mathfrak{r}_{\prec}(y) \mathfrak{r}_{\prec}(x)\right) z .
\end{aligned}
$$


Similarly, $\left(\mathfrak{l}_{\succ}, \mathfrak{r}_{\prec}\right)$ satisfies the other axioms defining a bimodule of $(\operatorname{Alt}(A), \circ)$.

Let $(A, \prec, \succ)$ be a prealternative algebra. Then by Propositions 2.4 and 2.9, both $\left(A^{*}, \mathfrak{r}_{\circ}^{*}, \mathfrak{l}_{\circ}^{*}\right)$ and $\left(A^{*}, \mathfrak{r}_{<}^{*}, \mathfrak{l}_{\succ}^{*}\right)$ are bimodules of $\operatorname{Alt}(A)$.

The next definition is motivated by the notion of $\mathbb{C}$-operator as a generalization of (the operator form of) the classical Yang-Baxter equation in [Kupershmidt 1999]; see also [Bai 2007].

Definition 2.10. Let $(V, L, R)$ be a bimodule of an alternative algebra $(A, \circ)$. A linear map $T: V \rightarrow A$ is called an O-operator associated to $(V, L, R)$ if

$$
T(u) \circ T(v)=T(L(T(u)) v+R(T(v)) u) \quad \text { for all } u, v \in V .
$$

Proposition 2.11. Let $T: V \rightarrow A$ be an 0 -operator of an alternative algebra $(A, \circ)$ associated to a bimodule $(V, L, R)$. Then there exists a prealternative algebra structure on $V$ given by

$$
u \prec v=R(T(v)) u \quad \text { and } \quad u \succ v=L(T(u)) v \quad \text { for all } u, v \in V .
$$

Therefore $V$ is an alternative algebra as the associated alternative algebra of this prealternative algebra and $T$ is a homomorphism of alternative algebras. Furthermore, $T(V)=\{T(v) \mid v \in V\} \subset A$ is an alternative subalgebra of $(A, \circ)$ and there is an induced prealternative algebra structure on $T(V)$ given by

$$
T(u) \prec T(v)=T(u \prec v) \quad \text { and } \quad T(u) \succ T(v)=T(u \succ v) \quad \text { for all } u, v \in V .
$$

Moreover, the associated alternative algebra structure is just the alternative subalgebra structure of $(A, \circ)$ and $T$ is a homomorphism of prealternative algebras.

Proof. We only prove one identity, with $(V, \prec, \succ)$ being a prealternative algebra as an example. The proof of the others is similar. For any $u, v, w \in V$,

$$
\begin{aligned}
(u \succ v) \prec w+(v \prec u) \prec w & =R(T(w)) L(T(u)) v+R(T(w)) R(T(u)) v, \\
u \succ(v \prec w)+v \prec(u \circ v) & =L(T(u)) R(T(w)) v+R(T(u \circ w)) v .
\end{aligned}
$$

By (2-3), (2-7) and (2-8), we show that

$(u, v, w)_{m}+(v, u, w)_{r}=(u \succ v) \prec w+(v \prec u) \prec w-u \succ(v \prec w)-v \prec(u \circ v)=0$.

The remaining parts of the conclusion are obvious.

Definition 2.12 [Schafer 1952]. Let $(A, \circ)$ be an alternative algebra and $(V, L, R)$ be a bimodule. A 1-cocycle of $A$ into $V$ is a linear map $D: A \rightarrow V$ satisfying

$$
D(x \circ y)=L(x) D(y)+R(y) D(x) \text { for all } x, y \in A .
$$

Proposition 2.13. For $(A, \circ)$ an alternative algebra, the following conditions are equivalent. 
(1) There is a compatible prealternative algebra structure $(A, \prec, \succ)$ on $(A, \circ)$.

(2) There is an invertible 0-operator.

(3) There is a bijective 1-cocycle.

Proof. (3) implies (2). If $D$ is a bijective 1-cocycle of $(A, \circ)$ into a bimodule $(V, L, R)$, then $D^{-1}$ is an 0 -operator associated to $(V, L, R)$.

(2) implies (1). If $T: V \rightarrow A$ is an invertible 0 -operator associated to a bimodule $(V, L, R)$, then there is a compatible prealternative algebra structure on $A$ given by $x \prec y=T\left(R(y) T^{-1}(x)\right)$ and $x \succ y=T\left(L(x) T^{-1}(y)\right)$ for all $x, y \in A$.

(1) implies (3). If $(A, \prec, \succ)$ is a compatible prealternative algebra structure on $(A, \circ)$, then it is obvious that the identity map id is a bijective 1-cocycle of $A$ into the bimodule $\left(A, \mathfrak{l}_{\succ}, \mathfrak{r}_{\prec}\right)$.

Example 2.14. Let $(A, \circ)$ be an alternative algebra graded by positive integers, that is, $A=\bigoplus_{i \in \mathbb{N}} A_{i}$ and $A_{i} \circ A_{j} \subset A_{i+j}$. Then there is a bijective 1-cocycle associated to the bimodule $\left(A, \mathfrak{l}_{\circ}, \mathfrak{r}_{\circ}\right)$ defined by $D\left(x_{i}\right)=i x_{i}$ for $x_{i} \in A_{i}$. Therefore there exists a compatible prealternative algebra structure on $(A, \circ)$ given by

$$
x_{i} \succ x_{j}=\frac{j}{i+j} x_{i} \circ x_{j} \quad \text { and } \quad x_{i} \prec x_{j}=\frac{i}{i+j} x_{i} \circ x_{j} \quad \text { for all } x_{i} \in A_{i}, x_{j} \in A_{j} .
$$

Definition 2.15. Let $(A, \circ)$ be an arbitrary algebra (not necessarily associative) and $\omega$ be a skew-symmetric bilinear form on $A$. The bilinear form $\omega$ is said to be closed if $\omega$ satisfies

$$
\omega(a \circ b, c)+\omega(b \circ c, a)+\omega(c \circ a, b)=0 \quad \text { for all } a, b, c \in A .
$$

If $\omega$ is also nondegenerate, then $\omega$ is said to be symplectic. An alternative algebra $A$ equipped with a symplectic form is called a symplectic alternative algebra.

Proposition 2.16. Let $(A, \circ, \omega)$ be an alternative algebra with symplectic form $\omega$. Then A has a compatible prealternative algebra structure $\prec, \succ$ given by

$$
\omega(x \prec y, z)=\omega(x, y \circ z) \quad \text { and } \quad \omega(x \succ y, z)=\omega(y, z \circ x) \quad \text { for all } x, y, z \in A \text {. }
$$

Proof. Define a linear map $T: A \rightarrow A^{*}$ by $\langle T(x), y\rangle=\omega(x, y)$ for all $x, y \in A$. Then $T$ is invertible and $T$ is a 1-cocycle of $A$ into the bimodule $\left(A^{*}, \mathfrak{r}_{\circ}^{*}, \mathfrak{l}_{\circ}^{*}\right)$. So by Proposition 2.13, there is a compatible prealternative algebra structure $\prec, \succ$ on $A$ given by

$$
x \prec y=T^{-1}\left(\mathfrak{l}_{\circ}^{*}(y) T(x)\right) \quad \text { and } \quad x \succ y=T^{-1}\left(\mathfrak{r}_{\circ}^{*}(x) T(y)\right) \text { for all } x, y \in A .
$$

Thus, for any $x, y \in A$,

$$
\begin{aligned}
& \omega(x \prec y, z)=\langle T(x \prec y), z\rangle=\left\langle\mathfrak{l}_{\circ}^{*}(y) T(x), z\right\rangle=\omega(x, y \circ z), \\
& \omega(x \succ y, z)=\langle T(x \succ y), z\rangle=\left\langle\mathfrak{r}_{\circ}^{*}(x) T(y), z\right\rangle=\omega(y, z \circ x) .
\end{aligned}
$$




\section{Alternative D-bialgebras and an alternative analogue of the classical Yang-Baxter equation}

Definition 3.1 [Goncharov 2007; Zhelyabin 1997]. Let $M$ be an arbitrary variety of $\mathbf{k}$-algebras and $(A, \circ)$ be an algebra in $M$ with comultiplication $\triangle$. Then $(A, \circ, \triangle)$ is called an M-bialgebra in the sense of Drinfeld if $D(A)$ belongs to $M$, where $D(A)=A \oplus A^{*}$ is equipped with the multiplication

$$
\begin{aligned}
(a+f) \star(b+g)=(a \circ b+f \cdot b+a \cdot g)+ & (f * g+f \bullet b+a \bullet g) \\
& \text { for all } a, b \in A \text { and } f, g \in A^{*},
\end{aligned}
$$

where

$$
\begin{array}{ll}
f \cdot a & =\sum_{a} a_{(1)}\left\langle f, a_{(2)}\right\rangle, \quad\langle f \bullet a, b\rangle=\langle f a \circ b\rangle, \\
a \cdot f=\sum_{a}\left\langle f, a_{(1)}\right\rangle a_{(2)}, & \langle a \bullet f, b\rangle=\langle f, b \circ a\rangle, \\
\triangle(a) & =\sum_{a} a_{(1)} \otimes a_{(2)},
\end{array}
$$

and the multiplication $*$ on $A^{*}$ is induced by $\triangle$. In this case, $D(A)=A \oplus A^{*}$ is called the Drinfeld double of $A$. In particular, when $M$ is a variety of alternative algebras, $(A, \circ, \triangle)$ is called an alternative D-bialgebra.

Remark 3.2. Goncharov [2007] notes that an alternative $D$-bialgebra $(A, \circ, \Delta)$ is equivalent to an alternative analogue of Manin triple [Chari and Pressley 1994]: There is an alternative algebra structure on the direct sum $A \oplus A^{*}$ of the underlying vector spaces of $A$ and $A^{*}$ such that both $A$ and $A^{*}$ are subalgebras and the symmetric bilinear form on $A \oplus A^{*}$ given by

$$
\mathscr{B}\left(x+a^{*}, y+b^{*}\right)=\left\langle a^{*}, y\right\rangle+\left\langle x, b^{*}\right\rangle \text { for all } x, y \in A \text { and } a^{*}, b^{*} \in A^{*}
$$

is invariant. Recall that a bilinear form $\mathscr{B}$ on an alternative algebra $(A, \circ)$ is called invariant if

$$
\mathscr{B}(x \circ y, z)=\mathscr{B}(x, y \circ z) \text { for all } x, y, z \in A .
$$

It is easy to show that $(A, \circ, \Delta)$ being an alternative $D$-bialgebra is equivalent to $\left(A, A^{*}, \mathfrak{r}_{\circ}^{*}, \mathfrak{l}_{\circ}^{*}, \mathfrak{r}_{*}^{*}, \mathfrak{l}_{*}^{*}\right)$ being a matched pair of alternative algebras in the sense of Proposition 4.7.

Definition 3.3. Let $(A, \circ)$ be an alternative algebra and $r=\sum_{i} a_{i} \otimes b_{i} \in A \otimes A$. Then the pair $(A, r)$ is called a coboundary alternative D-bialgebra if $\left(A, \circ, \triangle_{r}\right)$, where

$$
\triangle_{r}(x)=\sum_{i} a_{i} \circ x \otimes b_{i}-\sum_{i} a_{i} \otimes x \circ b_{i} \quad \text { for all } x \in A
$$

is an alternative $D$-bialgebra. 
Theorem 3.4 [Goncharov 2007]. Let $(A, \circ)$ be an alternative algebra and let $r \in A \otimes A$. Assume that $r$ is skew-symmetric and

$$
C_{A}(r)=r_{23} \circ r_{12}-r_{12} \circ r_{13}-r_{13} \circ r_{23}=0 .
$$

Then $\left(A, \circ, \triangle_{r}\right)$ is an alternative D-bialgebra.

Definition 3.5. Let $(A, \circ)$ be an alternative algebra and let $r \in A \otimes A$. Goncharov [2007] calls Equation (3-5) an alternative analogue of the classical Yang-Baxter equation. We also call it the alternative Yang-Baxter equation in $(A, \circ)$.

Proposition 3.6. Let $(A, \circ)$ be an alternative algebra and $r \in A \otimes A$. Then $r$ is a skew-symmetric solution of the alternative Yang-Baxter equation in $(A, \circ)$ if and only if $T_{r}$ is an 0-operator associated to the bimodule $\left(A^{*}, \mathfrak{r}_{\circ}^{*}, \mathfrak{l}_{\circ}^{*}\right)$, that is, $T_{r}$ satisfies the equation

$$
T_{r}\left(a^{*}\right) \circ T_{r}\left(b^{*}\right)=T_{r}\left(\mathfrak{r}_{\circ}^{*}\left(T_{r}\left(a^{*}\right)\right) b^{*}+\mathfrak{l}_{\circ}^{*}\left(T_{r}\left(b^{*}\right)\right) a^{*}\right) \quad \text { for all } a^{*}, b^{*} \in A^{*} .
$$

So there is a prealternative algebra structure on $A^{*}$ given by

$$
a^{*} \prec b^{*}=\mathfrak{l}_{\circ}^{*}\left(T_{r}\left(b^{*}\right)\right) a^{*} \quad \text { and } \quad a^{*} \succ b^{*}=\mathfrak{r}_{\circ}^{*}\left(T_{r}\left(a^{*}\right)\right) b^{*} \quad \text { for all } a^{*}, b^{*} \in A^{*} .
$$

Moreover, the associated alternative algebra structure is exactly the alternative algebra structure on $A^{*}$ as a subalgebra of $D(A)=A \oplus A^{*}$ that is induced from the comultiplication defined by (3-4). We denote this alternative algebra structure on $A^{*}$ by $A^{*}(r)$.

Proof. Let $\left\{e_{i}, \ldots, e_{n}\right\}$ be a basis of $A$ and $\left\{e_{i}^{*}, \ldots, e_{n}^{*}\right\}$ its dual. Suppose that $e_{i} \circ e_{j}=\sum_{k} c_{i j}^{k} e_{k}$ and $r=\sum_{i, j} a_{i j} e_{i} \otimes e_{j}$. Hence $a_{i j}=-a_{j i}$ and $T_{r}\left(e_{l}^{*}\right)=\sum_{k} a_{k l} e_{k}$. Then $r$ is a solution of the alternative Yang-Baxter equation in $(A, \circ)$ if and only if for any $i, k, t$

$$
\sum_{j s} a_{s t} a_{i j} c_{s j}^{k}-a_{j k} a_{s t} c_{j s}^{i}-a_{i j} a_{k s} c_{j s}^{t}=0 .
$$

The left hand side of this equation is precisely the coefficient of $e_{i}$ in

$$
-T_{r}\left(e_{k}^{*}\right) \circ T_{r}\left(e_{t}^{*}\right)+T_{r}\left(\mathfrak{r}_{\circ}^{*}\left(T_{r}\left(e_{k}^{*}\right)\right) e_{t}^{*}+\mathfrak{l}_{\circ}^{*}\left(T_{r}\left(e_{t}^{*}\right)\right) e_{k}^{*}\right) .
$$

Thus the first half part of the conclusion holds. It is easy to get the other results.

Corollary 3.7. Let $(A, \circ)$ be an alternative algebra and $r \in A \otimes A$. Assume $r$ is skew-symmetric and there exists a nondegenerate symmetric invariant bilinear form $\mathscr{B}$ on $(A, \circ)$. Define a linear map $\varphi: A \rightarrow A^{*}$ by $\langle\varphi(x), y\rangle=\mathscr{B}(x, y)$ for any $x, y \in A$. Then $r$ is a solution of the alternative Yang-Baxter equation in $(A, \circ)$ if and only if $\tilde{T}_{r}=T_{r} \varphi: A \rightarrow A$ is an 0 -operator associated to the bimodule $\left(A, \mathfrak{l}_{\circ}, \mathfrak{r}_{\circ}\right)$, that is, $\tilde{T}_{r}$ satisfies the equation

$$
\tilde{T}_{r}(x) \circ \tilde{T}_{r}(y)=\tilde{T}_{r}\left(\tilde{T}_{r}(x) \circ y+x \circ \tilde{T}_{r}(y)\right) \quad \text { for all } x, y \in A .
$$


Hence there is a prealternative algebra structure on A given by

$$
x \prec y=x \circ \tilde{T}_{r}(y) \text { and } x \succ y=\tilde{T}_{r}(x) \circ y \text { for all } x, y \in A .
$$

Proof. For all $x, y, z \in A$, we have

$$
\left\langle\varphi\left(\mathfrak{l}_{\circ}(x) y\right), z\right\rangle=\mathscr{B}(x \circ y, z)=\mathscr{B}(z, x \circ y)=\mathscr{B}(y, z \circ x)=\left\langle\mathfrak{r}_{\circ}^{*}(x) \varphi(y), z\right\rangle .
$$

Hence $\varphi\left(\mathfrak{l}_{\circ}(x) y\right)=\mathfrak{r}_{\circ}^{*}(x) \varphi(y)$ and similarly $\varphi\left(\mathfrak{r}_{\circ}(x) y\right)=\mathfrak{l}_{\circ}^{*}(x) \varphi(y)$ for any $x, y \in A$. Let $a^{*}=\varphi(x), b^{*}=\varphi(y)$. Then by Proposition 3.6, $r$ is a solution of the alternative Yang-Baxter equation in $(A, \circ)$ if and only if

$$
\begin{aligned}
T_{r} \varphi(x) \circ T_{r} \varphi(y)=T_{r}\left(a^{*}\right) \circ T_{r}\left(b^{*}\right) & =T_{r}\left(\mathfrak{r}_{\circ}^{*}\left(T_{r}\left(a^{*}\right)\right) b^{*}+\mathfrak{l}_{\circ}^{*}\left(T_{r}\left(b^{*}\right)\right) a^{*}\right) \\
& =T_{r} \varphi\left(T_{r} \varphi(x) \circ y+x \circ T_{r} \varphi(y)\right) .
\end{aligned}
$$

Remark 3.8. Equation (3-8) is exactly the Rota-Baxter relation of weight zero for an alternative algebra; see [Baxter 1960; Rota 1969].

Proposition 3.9. Let $(A, \circ)$ be an alternative algebra, $(V, L, R)$ a bimodule of $A$, and $\left(V^{*}, R^{*}, L^{*}\right)$ the dual bimodule. Let $T: V \rightarrow A$ be a linear map that can be identified as an element in $A \ltimes_{R^{*}, L^{*}} V^{*} \otimes A \ltimes R_{R^{*}, L^{*}} V^{*}$. Then $T$ is an $\mathbf{O}$-operator of $A$ associated to $(V, L, R)$ if and only if $r=T-\sigma(T)$ is a skew-symmetric solution of the alternative Yang-Baxter equation in $A \ltimes_{R^{*}, L^{*}} V^{*}$.

Proof. Let $\left\{e_{1}, \ldots, e_{n}\right\}$ be a basis of $A$. Let $\left\{v_{1}, \ldots, v_{m}\right\}$ be a basis of $V$ and $\left\{v_{1}^{*}, \ldots, v_{m}^{*}\right\}$ be its dual. Set $T\left(v_{i}\right)=\sum_{k=1}^{n} a_{i k} e_{k}$ for $i=1, \ldots, m$. Then

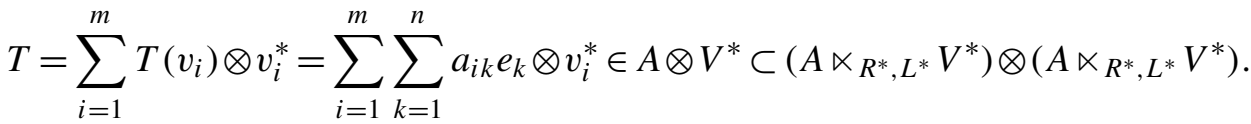

Therefore we have

$$
\begin{array}{r}
r_{12} \circ r_{13}=\sum_{i, k=1}^{m}\left(T\left(v_{i}\right) \circ T\left(v_{k}\right) \otimes v_{i}^{*} \otimes v_{k}^{*}-R^{*}\left(T\left(v_{i}\right)\right) v_{k}^{*} \otimes v_{i} \otimes T\left(v_{k}\right)\right. \\
\left.-L^{*}\left(T\left(v_{k}\right)\right) v_{i}^{*} \otimes T\left(v_{i}\right) \otimes v_{k}^{*}\right),
\end{array}
$$

$r_{23} \circ r_{12}=\sum_{i, j=1}^{m}\left(T\left(v_{k}\right) \otimes R^{*}\left(T\left(v_{i}\right)\right) v_{k}^{*} \otimes v_{i}^{*}-v_{k}^{*} \otimes T\left(v_{i}\right) \circ T\left(v_{k}\right) \otimes v_{i}^{*}\right.$ $\left.+v_{k}^{*} \otimes L^{*}\left(T\left(v_{k}\right)\right) v_{i}^{*} \otimes T\left(v_{i}\right)\right)$,

$r_{13} \circ r_{23}=\sum_{i, k=1}^{m}\left(v_{i}^{*} \otimes v_{k}^{*} \otimes T\left(v_{i}\right) \circ T\left(v_{k}\right)-T\left(v_{i}\right) \otimes v_{k}^{*} \otimes L^{*}\left(T\left(v_{k}\right)\right) v_{i}^{*}\right.$ $\left.-v_{i}^{*} \otimes T\left(v_{k}\right) \otimes R^{*}\left(T\left(v_{i}\right)\right) v_{k}^{*}\right)$.

By the definition of a dual bimodule, we know $L^{*}\left(T\left(v_{k}\right)\right) v_{i}^{*}=\sum_{j=1}^{m}\left\langle v_{i}^{*}, L\left(T\left(v_{k}\right)\right) v_{j}\right\rangle v_{j}^{*}, \quad R^{*}\left(T\left(v_{k}\right)\right) v_{i}^{*}=\sum_{j=1}^{m}\left\langle v_{i}^{*}, R\left(T\left(v_{k}\right)\right) v_{j}\right\rangle v_{j}^{*}$. 
Then

$$
\begin{aligned}
\sum_{i, k=1}^{m} T\left(v_{i}\right) \otimes v_{k}^{*} \otimes L^{*}\left(T\left(v_{k}\right)\right) v_{i}^{*} & =\sum_{i, k=1}^{m} \sum_{j=1}^{m}\left\langle v_{j}^{*}, L\left(T\left(v_{k}\right)\right) v_{i}\right\rangle T\left(v_{j}\right) \otimes v_{k}^{*} \otimes v_{i}^{*} \\
& =\sum_{i, k=1}^{m} T\left(\left\langle v_{j}^{*}, L\left(T\left(v_{k}\right)\right) v_{i}\right\rangle v_{j}\right) \otimes v_{k}^{*} \otimes v_{i}^{*} \\
& =\sum_{i, k=1}^{m} T\left(L\left(T\left(v_{k}\right)\right) v_{i}\right) \otimes v_{k}^{*} \otimes v_{i}^{*} .
\end{aligned}
$$

Hence, we get

$$
\begin{aligned}
r_{12} \circ r_{13}+r_{13} \circ & r_{23}-r_{23} \circ r_{12} \\
=\sum_{i, k=1}^{m}( & \left(\begin{array}{l} 
\\
\left.\quad\left(v_{i}\right) \circ T\left(v_{k}\right)-T\left(L\left(T\left(v_{i}\right)\right) v_{k}\right)-T\left(R\left(T\left(v_{k}\right)\right) v_{i}\right)\right) \otimes v_{i}^{*} \otimes v_{k}^{*} \\
\quad+v_{k}^{*} \otimes\left(T\left(v_{i}\right) \circ T\left(v_{k}\right)-T\left(L\left(T\left(v_{i}\right)\right) v_{k}\right)-T\left(R\left(T\left(v_{k}\right)\right) v_{i}\right)\right) \otimes v_{i}^{*} \\
\left.\quad+v_{i}^{*} \otimes v_{k}^{*} \otimes\left(T\left(v_{i}\right) \circ T\left(v_{k}\right)-T\left(L\left(T\left(v_{i}\right)\right) v_{k}\right)-T\left(R\left(T\left(v_{k}\right)\right) v_{i}\right)\right)\right) .
\end{array}\right.
\end{aligned}
$$

So $r$ is a solution of the alternative Yang-Baxter equation in $A \ltimes R^{*}, L^{*} V^{*}$ if and only if $T$ is an 0 -operator of $A$ associated to $(V, L, R)$.

Proposition 3.10 [Goncharov 2007]. Let $(A, \circ)$ be an alternative algebra and let $r \in A \otimes A$. Suppose that $r$ is skew-symmetric and nondegenerate. Then $r$ is a solution of the alternative Yang-Baxter equation in $A$ if and only if the bilinear form $\omega$ induced by $r$ through (1-8) is a symplectic form.

Corollary 3.11. Let $(A, \prec, \succ)$ be a prealternative algebra. Let $\left\{e_{1}, \ldots, e_{n}\right\}$ be a basis of $A$ and let $\left\{e_{1}^{*}, \ldots, e_{n}^{*}\right\}$ be its dual. Then $r=\sum_{i}\left(e_{i} \otimes e_{i}^{*}-e_{i}^{*} \otimes e_{i}\right)$ is a nondegenerate solution of the alternative Yang-Baxter equation in $\operatorname{Alt}(A) \ltimes_{\mathfrak{r}_{<}^{*},{ }_{*}^{*}} A^{*}$. The symplectic form $\omega_{p}$ induced by $r$ through (1-8) is given by

(3-10) $\omega_{p}\left(x+a^{*}, y+b^{*}\right)=\left\langle a^{*}, y\right\rangle-\left\langle x, b^{*}\right\rangle$ for all $x, y \in A$ and $a^{*}, b^{*} \in A^{*}$.

Proof. It follows from the fact that $T=\mathrm{id}$ is an $\mathrm{O}$-operator of $\operatorname{Alt}(A)$ associated to the bimodule $\left(A, \mathfrak{l}_{\succ}, \mathfrak{r}_{\prec}\right)$.

Proposition 3.12. Let $(A, \circ, \omega)$ be an alternative algebra with symplectic form $\omega$. Suppose that there is a compatible prealternative algebra structure $\prec, \succ$ on $A$ given by Proposition 2.16 and a prealternative algebra structure $\prec_{*}, \succ_{*}$ on $A^{*}$ given by (3-7), where the solution $r$ of the alternative Yang-Baxter equation in $(A, \circ)$ is induced by $\omega$ through (1-8). Let $a^{*} * b^{*}=a^{*} \prec_{*} b^{*}+a^{*} \succ_{*} b^{*}$ for any $a^{*}, b^{*} \in A^{*}$. Then there is a prealternative algebra structure $\prec_{0}, \succ_{0}$ on $A \oplus A^{*}$ given for any $x, y \in A$ and $a^{*}, b^{*} \in A^{*}$ by

$$
\begin{aligned}
& \left(x, a^{*}\right) \prec_{0}\left(y, b^{*}\right)=\left(x \prec y+\mathfrak{r}_{*}^{*}\left(b^{*}\right) x, a^{*} \prec_{*} b^{*}+\mathfrak{r}_{\circ}^{*}(y) a^{*}\right), \\
& \left(x, a^{*}\right) \succ_{0}\left(y, b^{*}\right)=\left(x \succ y+\mathfrak{r}_{*}^{*}\left(a^{*}\right) y, a^{*} \succ_{*} b^{*}+\mathfrak{r}_{\circ}^{*}(x) b^{*}\right) .
\end{aligned}
$$


Moreover, the associated alternative algebra is just the Drinfeld double $D(A)$ for the coboundary alternative D-bialgebra $\left(A, \circ, \Delta_{r}\right)$.

Proof. In fact, since $r$ is invertible, it is easy to show for any $x, y \in A$ and $a^{*}, b^{*} \in A^{*}$ that

$$
\begin{aligned}
\mathfrak{l}_{*}^{*}\left(b^{*}\right) x=x \prec T_{r}\left(b^{*}\right), & \mathfrak{l}_{\circ}^{*}(y) a^{*}=a \prec_{*} T_{r}^{-1}(y), \\
\mathfrak{r}_{*}^{*}\left(a^{*}\right) y=T_{r}\left(a^{*}\right) \succ y, & \mathfrak{r}_{\circ}^{*}(x) b^{*}=T_{r}^{-1}(x) \succ_{*} b^{*} .
\end{aligned}
$$

So for any $z \in A$ and $c^{*} \in A^{*}$,

$$
\begin{aligned}
&\left(\left(x, a^{*}\right) \succ_{0}\left(y, b^{*}\right)\right) \prec_{0}\left(z, c^{*}\right) \\
&=\left(\left(x+T_{r}\left(a^{*}\right)\right) \succ y,\left(T_{r}^{-1}(x)+a^{*}\right) \succ_{*} b^{*}\right) \prec_{0}\left(z, c^{*}\right) \\
&=\left((x \succ y) \prec z+(x \succ y) \prec T_{r}\left(c^{*}\right)+\left(T_{r}\left(a^{*}\right) \succ y\right) \prec z+\left(T_{r}\left(a^{*}\right) \succ y\right) \prec T_{r}\left(c^{*}\right),\right. \\
&\left(T_{r}^{-1}(x) \succ_{*} b^{*}\right) \prec_{*} T_{r}^{-1}(z)+\left(T_{r}^{-1}(x) \succ_{*} b^{*}\right) \prec_{*} c^{*} \\
&\left.+\left(a^{*} \succ b^{*}\right) \prec_{*} T_{r}^{-1}(z)+\left(a^{*} \succ_{*} b^{*}\right) \prec_{*} c^{*}\right) .
\end{aligned}
$$

Similarly,

$$
\begin{aligned}
& \left(\left(y, b^{*}\right) \prec_{0}\left(x, a^{*}\right)\right) \prec_{0}\left(z, c^{*}\right) \\
& =\left((y \prec x) \prec z+(y \prec x) \prec T_{r}\left(c^{*}\right)+\left(y \prec T_{r}\left(a^{*}\right)\right) \prec z+\left(y \prec T_{r}\left(a^{*}\right)\right) \prec T_{r}\left(c^{*}\right),\right. \\
& \left(b^{*} \prec_{*} T_{r}^{-1}(x)\right) \prec_{*} T_{r}^{-1}(z)+\left(b^{*} \prec_{*} a^{*}\right) \prec_{*} T_{r}^{-1}(z) \\
& \left.+\left(b^{*} \prec_{*} T_{r}^{-1}(x)\right) \prec_{*} c^{*}+\left(b^{*} \prec_{*} a^{*}\right) \prec_{*} c^{*}\right), \\
& \left(x, a^{*}\right) \succ_{0}\left(\left(y, b^{*}\right) \prec_{0}\left(z, c^{*}\right)\right) \\
& =\left(x \succ\left(y \prec T_{r}\left(c^{*}\right)\right)+x \succ(y \prec z)+T_{r}\left(a^{*}\right) \succ(y \prec z)+T_{r}\left(a^{*}\right) \succ\left(y \prec T_{r}\left(c^{*}\right)\right),\right. \\
& T_{r}^{-1}(x) \succ_{*}\left(b^{*} \prec_{*} c^{*}\right)+T_{r}^{-1}(x) \succ_{*}\left(b^{*} \prec_{*} T_{r}^{-1}(z)\right) \\
& \left.+a^{*} \succ_{*}\left(b^{*} \prec_{*} c^{*}\right)+a^{*} \succ_{*}\left(b^{*} \prec_{*} T_{r}^{-1}(z)\right)\right) \text {, } \\
& \left(y, b^{*}\right) \prec_{*}\left(\left(x, a^{*}\right) \bullet\left(z, c^{*}\right)\right) \\
& =\left(y \prec\left(T_{r}\left(a^{*}\right) \circ T_{r}\left(c^{*}\right)\right)+y \prec\left(T_{r}\left(a^{*}\right) \prec z\right)+y \prec\left(x \succ T_{r}\left(c^{*}\right)\right)\right. \\
& +y \prec(x \circ z)+y \prec\left(x \prec T_{r}\left(c^{*}\right)\right)+y \prec\left(T_{r}\left(a^{*}\right) \succ z\right), b^{*} \prec_{*}\left(a^{*} * c^{*}\right) \\
& +b^{*} \prec_{*}\left(a^{*} \prec_{*} T_{r}^{-1}(z)\right)+b^{*} \prec_{*}\left(T_{r}^{-1}(x) \succ_{*} c^{*}\right)+b^{*} \prec_{*}\left(T_{r}^{-1}(x) * T_{r}^{-1}(z)\right) \\
& \left.+b^{*} \prec_{*}\left(T_{r}^{-1}(x) \prec_{*} c^{*}\right)+b^{*} \prec_{*}\left(a^{*} \succ_{*} T_{r}^{-1}(z)\right)\right),
\end{aligned}
$$

where $\bullet=\prec_{0}+\succ_{0}$. Hence

$$
\begin{aligned}
\left(\left(x, a^{*}\right) \succ_{0}\left(y, b^{*}\right)\right) & \prec_{0}\left(z, c^{*}\right)+\left(\left(y, b^{*}\right) \prec_{0}\left(x, a^{*}\right)\right) \prec_{0}\left(z, c^{*}\right) \\
= & \left(x, a^{*}\right) \succ_{0}\left(\left(y, b^{*}\right) \prec_{0}\left(z, c^{*}\right)\right)+\left(y, b^{*}\right) \prec_{*}\left(\left(x, a^{*}\right) \bullet\left(z, c^{*}\right)\right) .
\end{aligned}
$$

Using a similar argument, we can prove that $\left(\prec_{0}, \succ_{0}\right)$ also satisfies (2-6) and the second equation in (2-5). 
Proposition 3.13. Let $(A, \circ)$ be an alternative algebra.

(1) For any skew-symmetric solution $r$ of the alternative Yang-Baxter equation, the Drinfeld double $D(A)$ of the coboundary alternative D-bialgebra $\left(A, \circ, \Delta_{r}\right)$ is isomorphic to $A \ltimes_{\mathfrak{r}_{\circ}^{*}, l_{0}^{*}} A^{*}$ as alternative algebras.

(2) The skew-symmetric solutions of the alternative Yang-Baxter equation in $(A, \circ)$ are in one-to-one correspondence with the linear maps $T_{r}: A^{*} \rightarrow A$ whose graphs

$$
\operatorname{graph}\left(T_{r}\right)=\left\{\left(T_{r}\left(a^{*}\right), a^{*}\right) \in A \ltimes_{\mathfrak{r}_{\circ}^{*},{ }_{\circ}^{*}} A^{*} \mid a^{*} \in A^{*}\right\}
$$

are Lagrangian subalgebras of $A \ltimes_{\mathfrak{r}_{\circ}^{*}, l_{0}^{*}} A^{*}$ with respect to the bilinear form given by (3-2). Consequently every alternative subalgebra that is also a Lagrangian $\operatorname{graph}\left(T_{r}\right)$ of $A \ltimes_{\mathfrak{r}_{0}^{*}, l_{0}^{*}} A^{*}$ carries a prealternative algebra structure defined for any $a^{*}, b^{*} \in A^{*}$ by

$$
\begin{aligned}
& \left(T_{r}\left(a^{*}\right), a^{*}\right) \prec\left(T_{r}\left(b^{*}\right), b^{*}\right)=\left(T_{r}\left(r_{\circ}^{*}\left(T_{r}\left(b^{*}\right)\right) a^{*}\right), \mathfrak{r}_{\circ}^{*}\left(T_{r}\left(b^{*}\right)\right) a^{*}\right), \\
& \left(T_{r}\left(a^{*}\right), a^{*}\right) \succ\left(T_{r}\left(b^{*}\right), b^{*}\right)=\left(T_{r}\left(\mathfrak{r}_{\circ}^{*}\left(T_{r}\left(a^{*}\right)\right) b^{*}\right), \mathfrak{r}_{\circ}^{*}\left(T_{r}\left(a^{*}\right)\right) b^{*}\right) .
\end{aligned}
$$

Proof. (1) Let $r$ be a skew-symmetric solution of the alternative Yang-Baxter equation in $(A, \circ)$. Let the operation in $A^{*}(r)$ be $*$. Then by Proposition 3.6, we know $a^{*} * b^{*}=\mathfrak{r}_{\circ}^{*}\left(T_{r}\left(a^{*}\right)\right) b^{*}+\mathfrak{r}_{\circ}^{*}\left(T_{r}\left(b^{*}\right) a^{*}\right)$ for any $a^{*}, b^{*} \in A^{*}$. We claim that for any $x, y \in A$ and $a^{*}, b^{*} \in A^{*}$, we have

$$
\mathfrak{r}_{*}^{*}\left(a^{*}\right) y+\mathfrak{l}_{*}^{*}\left(b^{*}\right) x=T_{r}\left(a^{*}\right) \circ y+x \circ T_{r}\left(b^{*}\right)-T_{r}\left(\mathfrak{r}_{\circ}^{*}(x) b^{*}+\mathfrak{r}_{\circ}^{*}(y) a^{*}\right) .
$$

In fact, it follows from the computation (for any $c^{*} \in A^{*}$ )

$$
\begin{aligned}
\left\langle\mathfrak{r}_{*}^{*}\left(a^{*}\right) y\right. & \left.+\mathfrak{l}_{*}^{*}\left(b^{*}\right) x, c^{*}\right\rangle=\left\langle y, c^{*} * a^{*}\right\rangle+\left\langle x, b^{*} * c^{*}\right\rangle \\
& =\left\langle y, \mathfrak{r}_{\circ}^{*}\left(T_{r}\left(c^{*}\right)\right) a^{*}+\mathfrak{l}_{\circ}^{*}\left(T_{r}\left(a^{*}\right)\right) c^{*}\right\rangle+\left\langle x, \mathfrak{r}_{\circ}^{*}\left(T_{r}\left(b^{*}\right)\right) c^{*}+\mathfrak{l}_{\circ}^{*}\left(T_{r}\left(c^{*}\right)\right) b^{*}\right\rangle \\
& =\left\langle y \circ T_{r}\left(c^{*}\right), a^{*}\right\rangle+\left\langle T_{r}\left(a^{*}\right) \circ y, c^{*}\right\rangle+\left\langle x \circ T_{r}\left(b^{*}\right), c^{*}\right\rangle+\left\langle T_{r}\left(c^{*}\right) \circ x, b^{*}\right\rangle \\
& =\left\langle T_{r}\left(a^{*}\right) \circ y+x \circ T_{r}\left(b^{*}\right)-T_{r}\left(\mathfrak{r}_{\circ}^{*}(x) b^{*}+\mathfrak{l}_{\circ}^{*}(y) a^{*}\right), c^{*}\right\rangle,
\end{aligned}
$$

where we use that $\left\langle T_{r}\left(a^{*}\right), b^{*}\right\rangle=-\left\langle a^{*}, T_{r}\left(b^{*}\right)\right\rangle$, which follows from the fact that $r$ is skew-symmetric. Define a linear map $\lambda:\left(D(A)=A \oplus A^{*}, \bullet\right) \rightarrow\left(A \ltimes_{\mathfrak{r}_{\circ}^{*}, *_{\circ}^{*}} A^{*}, \star\right)$ by $\lambda\left(\left(x, a^{*}\right)\right)=\left(T_{r}\left(a^{*}\right)+x, a^{*}\right)$ for all $x \in A, a^{*} \in A^{*}$. Then we have

$$
\begin{aligned}
\lambda\left(\left(x, a^{*}\right)\right) \star \lambda\left(\left(y, b^{*}\right)\right) & \\
= & \left(\left(T_{r}\left(a^{*}\right)+x\right) \circ\left(T_{r}\left(b^{*}\right)+y\right), \mathfrak{r}_{\circ}^{*}\left(T_{r}\left(a^{*}\right)+x\right) b^{*}+\mathfrak{l}_{\circ}^{*}\left(T_{r}\left(b^{*}\right)+x\right) a^{*}\right) \\
= & \left(T_{r}\left(a^{*} * b^{*}+\mathfrak{r}_{\circ}^{*}(x) b^{*}+\mathfrak{l}_{\circ}^{*}(y) a^{*}\right)+x \circ y+\mathfrak{r}_{*}^{*}\left(a^{*}\right) y+r_{*}^{*}\left(b^{*}\right) x, a^{*} * b^{*}\right. \\
& =\lambda\left(\left(x, a^{*}\right) \bullet\left(y, b^{*}\right)\right),
\end{aligned}
$$


where we used (3-6) and (3-11). Furthermore, it is easy to show that $\lambda$ is bijective. Therefore $\lambda$ is an isomorphism of alternative algebras.

(2) First, $\lambda\left(A^{*}(r)\right)=\operatorname{graph}\left(T_{r}\right)$. So $\operatorname{graph}\left(T_{r}\right)$ is a subalgebra of $A \ltimes_{\mathfrak{r}_{0}^{*}, l_{0}^{*}} A^{*}$. Since $r$ is skew-symmetric, $\operatorname{graph}\left(T_{r}\right)$ is isotropic with respect to the bilinear form defined by (3-2). Moreover, it has a complementary isotropic algebra $\lambda(A)=A$. So it is a Lagrangian subalgebra of $A \ltimes_{\mathfrak{r}_{0}^{*}, \psi_{0}^{*}} A^{*}$. Conversely, let $T: A^{*} \rightarrow A$ be a linear map whose graph $(T)$ is a Lagrangian subalgebra of $A \ltimes_{\mathfrak{r}_{0}^{*}, \text { I. }_{*}^{*}} A^{*}$. So $T$ is skew-symmetric, that is, $\left\langle T\left(a^{*}\right), b^{*}\right\rangle=-\left\langle T\left(b^{*}\right), a^{*}\right\rangle$ for any $a^{*}, b^{*} \in A^{*}$. Since $\operatorname{graph}(T)$ is a subalgebra, we have

$$
\begin{array}{r}
\left(T\left(a^{*}\right), a^{*}\right) \star\left(T\left(b^{*}\right), b^{*}\right)=\left(T\left(a^{*}\right) \circ T\left(b^{*}\right), \mathfrak{r}_{\circ}^{*}\left(T_{r}\left(a^{*}\right)\right) b^{*}+\mathfrak{l}_{\circ}^{*}\left(T_{r}\left(b^{*}\right)\right) a^{*}\right) \\
=\left(T_{r}\left(\mathfrak{r}_{\circ}^{*}\left(T_{r}\left(a^{*}\right)\right) b^{*}+\mathfrak{l}_{\circ}^{*}\left(T_{r}\left(b^{*}\right)\right) a^{*}\right), \mathfrak{r}_{\circ}^{*}\left(T_{r}\left(a^{*}\right)\right) b^{*}+\mathfrak{l}_{\circ}^{*}\left(T_{r}\left(b^{*}\right)\right) a^{*}\right) .
\end{array}
$$

Thus $T\left(a^{*}\right) \circ T\left(b^{*}\right)=T\left(\mathfrak{r}_{\circ}^{*}\left(T_{r}\left(a^{*}\right)\right) b^{*}+l_{\circ}^{*}\left(T_{r}\left(b^{*}\right)\right) a^{*}\right)$. By Proposition 3.6, $T$ corresponds to a skew-symmetric solution of the alternative Yang-Baxter equation in $(A, \circ)$. The last statement is obtained by transferring (by the isomorphism $\lambda$ ) the prealternative algebra structure of $A^{*}(r)$ to $\operatorname{graph}\left(T_{r}\right)$.

\section{Phase spaces of alternative algebras and matched pairs of alternative algebras}

Definition 4.1. Let $(A, \circ, \omega)$ be a symplectic alternative algebra. We call $A$ an $L$ symplectic alternative algebra if it is a direct sum of the underlying vector spaces of two Lagrangian subalgebras $A^{+}$and $A^{-}$, we denote it by $\left(A, \circ, A^{+}, A^{-}, \omega\right)$. Two $L$-symplectic alternative algebras $\left(A_{1}, \circ, A_{1}^{+}, A_{1}^{-}, \omega_{1}\right)$ and $\left(A_{2}, \circ, A_{2}^{+}, A_{2}^{-}, \omega_{2}\right)$ are isomorphic if there exists an isomorphism $\varphi: A_{1} \rightarrow A_{2}$ of alternative algebras such that, for all $a, b \in A_{1}$,

$$
\varphi\left(A_{1}^{+}\right)=A_{2}^{+}, \quad \varphi\left(A_{1}^{-}\right)=A_{2}^{-}, \quad \omega_{1}(a, b)=\varphi^{*} \omega_{2}(a, b)=\omega_{2}(\varphi(a), \varphi(b))
$$

It is straightforward to show that a symplectic alternative algebra $(A, \circ, \omega)$ is an $L$-symplectic alternative algebra if and only if $A$ is a direct sum of the underlying vector space of two isotropic subalgebras.

Proposition 4.2. Let $\left(A, \circ, A^{+}, A^{-}, \omega\right)$ be an L-symplectic alternative algebra. Then there exists a prealternative algebra structure on A given by Proposition 2.16 such that $A^{+}$and $A^{-}$are prealternative subalgebras. Two L-symplectic alternative algebras $\left(A_{i}, \circ, A_{i}^{+}, A_{i}^{-}, \omega_{i}\right)$ for $i=1,2$ are isomorphic if and only if there exists an isomorphism of prealternative algebras satisfying (4-1) in which the compatible prealternative algebras are given by Proposition 2.16.

Proof. If $a, b, c \in A^{+}$, then $\omega(a \prec b, c)=\omega(a, b \circ c)=0$. Since $A^{+}$is a Lagrangian subalgebra of $A$, we have $a \prec b \in A^{+}$for all $a, b \in A^{+}$. Similar arguments apply to $\succ$ and $A^{-}$. So the first conclusion holds. It is easy to get the second. 
Definition 4.3. Let $(A, \circ)$ be an alternative algebra. If there exists an alternative algebra structure on the direct sum of the underlying vector space of $A$ and $A^{*}$ such that $A$ and $A^{*}$ are alternative subalgebras and the natural skew-symmetric bilinear form $\omega_{p}$ on $A \oplus A^{*}$ given by (3-10) is a symplectic form, then it is called a phase space of the alternative algebra $A$.

Remark 4.4. The notion of phase space is borrowed from mathematical physics [Kupershmidt 1994; Bai 2006].

Proposition 4.5. Every L-symplectic alternative algebra $\left(A, \circ, A^{+}, A^{-}, \omega\right)$ is isomorphic to a phase space of $A^{+}$.

Proof. Since $A^{-}$and $\left(A^{+}\right)^{*}$ are identified by the symplectic form, we can transfer the alternative algebra structure on $A^{-}$to $\left(A^{+}\right)^{*}$. Hence the alternative algebra structure on $A^{+} \oplus A^{-}$can be transferred to $A^{+} \oplus\left(A^{+}\right)^{*}$.

Remark 4.6. By symmetry of $A^{+}$and $A^{-}$, every $L$-symplectic alternative algebra $\left(A, \circ, A^{+}, A^{-}, \omega\right)$ is isomorphic to a phase space of $A^{-}$.

Proposition 4.7. Let $(A, \circ)$ and $(B, *)$ be two alternative algebras. Suppose that there are linear maps $L_{A}, R_{A}: A \rightarrow \mathfrak{g l}(B)$ and $L_{B}, R_{B}: B \rightarrow \mathfrak{g l}(A)$ such that $\left(L_{A}, R_{A}\right)$ is a bimodule of $A$ and $\left(L_{B}, R_{B}\right)$ is a bimodule of $B$ and they satisfy the conditions

$$
\begin{aligned}
& L_{B}\left(\operatorname{Ass}_{A}(x) a\right) y+\left(\operatorname{Ass}_{B}(a) x\right) \circ y \\
& \quad=L_{B}(a)(x \circ y)+R_{B}\left(R_{A}(y) a\right) x+x \circ\left(L_{B}(a) y\right),
\end{aligned}
$$

(4-3) $\quad R_{B}(a)(x \circ y+y \circ x)$

$$
=R_{B}\left(L_{A}(y) a\right) x+x \circ\left(R_{B}(a) y\right)+R_{B}\left(L_{A}(x) a\right) y+y \circ\left(R_{B}(a) x\right),
$$

(4-4) $\quad R_{B}(a)(x \circ y)+L_{B}\left(L_{A}(x) a\right) y+\left(R_{B}(a) x\right) \circ y$

$$
=R_{B}\left(\operatorname{Ass}_{A}(y) a\right) x+x \circ\left(\operatorname{Ass}_{B}(a) y\right),
$$

(4-5) $\quad L_{B}(a)(x \circ y+y \circ x)$

$$
=\left(L_{B}(a) x\right) \circ y+L_{B}\left(R_{A}(x) a\right) y+\left(L_{B}(a) y\right) \circ x+L_{B}\left(R_{A}(y) a\right) x,
$$

(4-6) $\quad L_{A}\left(\operatorname{Ass}_{B}(a) x\right) b+\left(\operatorname{Ass}_{A}(x) a\right) * b$

$$
=L_{A}(x)(a * b)+R_{A}\left(R_{B}(b) x\right) a+a *\left(L_{A}(x) b\right),
$$

(4-7) $\quad R_{A}(x)(a * b+b * a)$

$$
=R_{A}\left(L_{B}(b) x\right) a+a *\left(R_{A}(x) b\right)+R_{A}\left(L_{B}(a) x\right) b+b *\left(R_{A}(x) a\right),
$$

(4-8) $\quad R_{A}(x)(a * b)+L_{A}\left(L_{B}(a) x\right) b+\left(R_{A}(x) a\right) * b$

$$
=R_{A}\left(\operatorname{Ass}_{B}(b) x\right) a+a *\left(\operatorname{Ass}_{A}(x) b\right),
$$

(4-9) $\quad L_{A}(x)(a * b+b * a)$

$$
=\left(L_{A}(x) a\right) * b+L_{A}\left(R_{B}(a) x\right) b+\left(L_{A}(x) b\right) * a+L_{A}\left(R_{B}(b) x\right) a,
$$


where $x, y \in A, a, b \in B$ and $\mathrm{Ass}_{i}=L_{i}+R_{i}$ for $i=A, B$. Then there is an alternative algebra structure on the vector space $A \oplus B$ given for all $x, y \in A$ and $a, b \in B$ by

$$
(x+a) \star(y+b)=\left(x \circ y+L_{B}(a) y+R_{B}(b) x\right)+\left(a * b+L_{A}(x) b+R_{A}(y) a\right)
$$

We denote this alternative algebra by $A \bowtie_{L_{A}, R_{A}}^{L_{B}, R_{B}} B$ or simply $A \bowtie B$. We call any ( $A, B, L_{A}, R_{A}, L_{B}, R_{B}$ ) satisfying the conditions above a matched pair of alternative algebras. Every alternative algebra that is a direct sum of the underlying vector spaces of two subalgebras can be obtained is this way.

Proof. Straightforward.

Proposition 4.8. Let $\left(A, \prec_{1}, \succ_{1}\right)$ be a prealternative algebra and $(\operatorname{Alt}(A), \circ)$ be the associated alternative algebra. Suppose there exists a prealternative algebra structure $\prec_{2}, \succ_{2}$ on the dual space $A^{*}$, with $\left(\operatorname{Alt}\left(A^{*}\right), *\right)$ the associated alternative algebra. Then there exists an L-symplectic alternative algebra structure on $A \oplus A^{*}$ such that $(\operatorname{Alt}(A), \circ)$ and $\left(\operatorname{Alt}\left(A^{*}\right), *\right)$ are Lagrangian subalgebras associated to the symplectic form (3-10) if and only if $\left(\operatorname{Alt}(A), \operatorname{Alt}\left(A^{*}\right), \mathfrak{r}_{\succ_{1}}^{*}, \mathfrak{l}_{\succ_{1}}^{*}, \mathfrak{r}_{\succ_{2}}^{*}, \mathfrak{l}_{\succ_{2}}^{*}\right)$ is a matched pair of alternative algebras. Every $L$-symplectic alternative algebra can be obtained in this way.

Proof. If $\left(\operatorname{Alt}(A), \operatorname{Alt}\left(A^{*}\right), \mathfrak{r}_{<_{1}}^{*}, \mathfrak{l}_{\succ_{1}}^{*}, \mathfrak{r}_{<_{2}}^{*}, l_{\succ_{2}}^{*}\right)$ is a matched pair of alternative algebras, then it is straightforward to show that the bilinear form (3-10) is a symplectic form of the alternative algebra $A_{\bowtie}:=\operatorname{Alt}(A) \bowtie_{\mathfrak{r}_{<1}^{*},}^{\mathfrak{r}_{2}^{*}, l_{\succ_{1}}^{*}, l^{*}} \operatorname{Alt}\left(A^{*}\right)$. Conversely, set $x \star a^{*}=L_{\circ}(x) a^{*}+R_{*}\left(a^{*}\right) x, a^{*} \star x=L_{*}\left(a^{*}\right) x+R_{\circ}(x) a^{*}$ for all $x \in A, a^{*} \in A^{*}$,

where $\star$ is the alternative algebra structure of $A_{\bowtie}$. Then $\left(A, A^{*}, L_{\circ}, R_{\circ}, L_{*}, R_{*}\right)$ is a matched pair of alternative algebras. Note that

$$
\begin{aligned}
\left\langle R_{\circ}(x) a^{*}, y\right\rangle & =\left\langle a^{*} \star x, y\right\rangle=-\omega_{p}\left(y, a^{*} \star x\right)=-\omega_{p}\left(x \succ_{1} y, a^{*}\right)=\left\langle r_{\succ_{1}}^{*}(x) a^{*}, y\right\rangle, \\
\left\langle L_{*}\left(a^{*}\right) x, b^{*}\right\rangle & =\left\langle a^{*} \star x, b^{*}\right\rangle=\omega_{p}\left(b^{*}, a^{*} \star x\right)=\omega_{p}\left(b^{*} \prec_{2} a^{*}, x\right)=\left\langle\mathfrak{r}_{\prec_{2}}^{*}\left(a^{*}\right) x, b^{*}\right\rangle,
\end{aligned}
$$

where $x, y \in A$ and $a^{*}, b^{*} \in A^{*}$. Hence, $R_{\circ}=\mathfrak{l}_{\succ_{1}}^{*}$ and $L_{*}=\mathfrak{r}_{\prec_{2}}^{*}$. Similarly, $L_{\circ}=\mathfrak{r}_{<_{1}}^{*}$ and $R_{*}=\mathfrak{l}_{\succ_{2}}^{*}$.

\section{Bimodules and matched pairs of prealternative algebras}

Definition 5.1. Let $(A, \prec, \succ)$ be a prealternative algebra and $V$ be a vector space. Let $L_{\prec}, R_{\prec}, L_{\succ}, R_{\succ}: A \rightarrow \mathfrak{g l}(V)$ be linear maps. We call $V$ (or $\left(L_{\prec}, R_{\prec}, L_{\succ}, R_{\succ}\right.$ ) or $\left.\left(V, L_{\prec}, R_{\prec}, L_{\succ}, R_{\succ}\right)\right)$ a representation or a bimodule of $A$ if (for any $x, y \in A$ )

$$
\begin{aligned}
L_{\succ}(x \circ y+y \circ x) & =L_{\succ}(x) L_{\succ}(y)+L_{\succ}(y) L_{\succ}(x), \\
R_{\succ}(y)\left(L_{\circ}(x)+R_{\circ}(x)\right) & =L_{\succ}(x) R_{\succ}(y)+R_{\succ}(x \succ y),
\end{aligned}
$$




$$
\begin{aligned}
R_{\prec}(y) L_{\succ}(x)+R_{\prec}(y) R_{\prec}(x) & =L_{\succ}(x) R_{\prec}(y)+R_{\prec}(x \circ y), \\
R_{\prec}(y) R_{\succ}(x)+R_{\prec}(y) L_{\prec}(x) & =R_{\succ}(x \prec y)+L_{\prec}(x) R_{\circ}(y), \\
L_{\prec}(x \succ y)+L_{\prec}(y \prec x) & =L_{\succ}(x) L_{\prec}(y)+L_{\prec}(y) L_{\circ}(x), \\
L_{\succ}(y \circ x)+R_{\prec}(x) L_{\succ}(y) & =L_{\succ}(y) L_{\succ}(x)+L_{\succ}(y) R_{\prec}(x), \\
R_{\succ}(y) R_{\circ}(x)+R_{\prec}(x) R_{\succ}(y) & =R_{\succ}(x \succ y)+R_{\succ}(y \prec x), \\
R_{\succ}(x) L_{\circ}(y)+L_{\prec}(y \succ x) & =L_{\succ}(y) R_{\succ}(x)+L_{\succ}(y) L_{\prec}(x), \\
R_{\prec}(y) R_{\prec}(x)+R_{\prec}(x) R_{\prec}(y) & =R_{\prec}(x \circ y+y \circ x), \\
R_{\prec}(y) L_{\prec}(x)+L_{\prec}(x \prec y) & =L_{\prec}(x)\left(R_{\circ}(y)+L_{\circ}(y)\right),
\end{aligned}
$$

where $x \circ y=x \prec y+x \succ y, L_{\circ}=L_{\succ}+L_{\prec}$ and $R_{\circ}=R_{\succ}+R_{\prec}$.

According to [Schafer 1995], $\left(V, L_{\prec}, R_{\prec}, L_{\succ}, R_{\succ}\right)$ is a bimodule of a prealternative algebra $(A, \prec, \succ)$ if and only if the direct sum $A \oplus V$ of vector spaces becomes a prealternative algebra (the semidirect sum) by defining multiplications in $A \oplus V$ for any $x, y \in A$ and $a, b \in V$ by

$$
\begin{aligned}
& (x+a) \prec(y+b)=x \prec y+L_{\prec}(x) b+R_{\prec}(y) a, \\
& (x+a) \succ(y+b)=x \succ y+L_{\succ}(x) b+R_{\succ}(y) a .
\end{aligned}
$$

We denote it by $A \ltimes_{L_{\prec}, R_{\prec}, L_{\succ}, R_{\succ}} V$ or simply $A \ltimes V$.

Proposition 5.2. Suppose $\left(V, L_{\prec}, R_{\prec}, L_{\succ}, R_{\succ}\right)$ is a bimodule of a prealternative algebra $(A, \prec, \succ)$. Let $(\operatorname{Alt}(A), \circ)$ be the associated alternative algebra.

(1) Both $\left(V, L_{\succ}, R_{\prec}\right)$ and $\left(V, L_{\circ}=L_{\prec}+L_{\succ}, R_{\circ}=R_{\prec}+R_{\succ}\right)$ are bimodules of $(\operatorname{Alt}(A), \circ)$.

(2) If $(V, L, R)$ is a bimodule of $(\operatorname{Alt}(A), \circ)$, then $(V, 0, R, L, 0)$ is a bimodule of $(A, \prec, \succ)$.

(3) $\left(V^{*},-R_{\succ}^{*}, L_{\circ}^{*}, R_{\circ}^{*},-L_{\prec}^{*}\right)$ is a bimodule of $(A, \prec, \succ)$.

Proof. We only prove (3) as an example. The others are straightforward. Since $\left(V, L_{\circ}, R_{\circ}\right)$ is a bimodule of $\operatorname{Alt}(A)$, we have $R_{\circ}\left(x^{2}\right)=R_{\circ}(x) R_{\circ}(x)$ for any $x \in A$. Hence $R_{\circ}(x \circ y+y \circ x)=R_{\circ}(x) R_{\circ}(y)+R_{\circ}(y) R_{\circ}(x)$ for all $x, y \in A$. So for any $v \in V$ and $u^{*} \in V^{*}$,

$$
\begin{aligned}
\left\langle R_{\circ}^{*}(x \circ y+y \circ x) u^{*}, v\right\rangle & =\left\langle u^{*}, R_{\circ}(x \circ y+y \circ x) v\right\rangle \\
& =\left\langle u^{*},\left(R_{\circ}(x) R_{\circ}(y)+R_{\circ}(y) R_{\circ}(x)\right) v\right\rangle \\
& =\left\langle\left(R_{\circ}^{*}(x) R_{\circ}^{*}(y)+R_{\circ}^{*}(y) R_{\circ}^{*}(x)\right) u^{*}, v\right\rangle .
\end{aligned}
$$

Therefore $R_{\circ}^{*}(x \circ y+y \circ x)=R_{\circ}^{*}(x) R_{\circ}^{*}(y)+R_{\circ}^{*}(y) R_{\circ}^{*}(x)$. Similarly, we can prove that $\left(-R_{\succ}^{*}, L_{\circ}^{*}, R_{\circ}^{*},-L_{\prec}^{*}\right)$ also satisfies the remaining requirements (5-2)-(5-10) of a bimodule. 
Example 5.3. Let $(A, \prec, \succ)$ be a prealternative algebra. Then $\left(\mathfrak{l}_{\prec}, \mathfrak{r}_{\prec}, \mathfrak{l}_{\succ}, \mathfrak{r}_{\succ}\right)$, $\left(0, \mathfrak{r}_{<}, \mathfrak{l}_{\succ}, 0\right),\left(0, \mathfrak{r}_{\circ}, \mathfrak{l}_{\circ}, 0\right),\left(0, \mathfrak{l}_{\circ}^{*}, \mathfrak{r}_{\circ}^{*}, 0\right),\left(0, \mathfrak{l}_{\succ}^{*}, \mathfrak{r}_{<}^{*}, 0\right)$ and $\left(-\mathfrak{r}_{\succ}^{*}, \mathfrak{l}_{\circ}^{*}, \mathfrak{r}_{\circ}^{*},-\mathfrak{l}_{<}^{*}\right)$ are bimodules of $(A, \prec, \succ)$.

Definition 5.4. Let $\left(A, \prec_{A}, \succ_{A}\right)$ and $\left(B, \prec_{B}, \succ_{B}\right)$ be two prealternative algebras. Suppose that there are linear maps

$$
L_{\prec_{A}}, R_{\succ_{A}}, L_{\succ_{A}}, R_{\succ_{A}}: A \rightarrow \mathfrak{g l}(B) \quad \text { and } \quad L_{\prec_{B}}, R_{\succ_{B}}, L_{\succ_{B}}, R_{\succ_{B}}: B \rightarrow \mathfrak{g l}(A)
$$

such that the products

$$
\begin{aligned}
& (x+a) \prec(y+b)=x \prec_{A} y+L_{\prec_{B}}(a) y+R_{\prec_{B}}(b) x+a \prec_{B} b+L_{\prec_{A}}(x) b+R_{\prec_{A}}(y) a, \\
& (x+a) \succ(y+b)=x \succ_{A} y+L_{\succ_{B}}(a) y+R_{\succ_{B}}(b) x+a \succ_{B} b+L_{\succ_{A}}(x) b+R_{\succ_{A}}(y) a,
\end{aligned}
$$

on the vector space $A \oplus B$ (for any $x, y \in A$ and $a, b \in B$ ) define a prealternative algebra structure. Then we call $\left(A, B, L_{\succ_{A}}, R_{\succ_{A}}, L_{\succ_{A}}, R_{\succ_{A}}, L_{\prec_{B}}, R_{\prec_{B}}\right.$, $L_{\succ_{B}}, R_{\succ_{B}}$ ) a matched pair of prealternative algebras, and we denote this pair by $A \bowtie_{L_{\succ_{A}}, R_{\succ_{A}}, L_{\succ_{A}}, R_{\succ_{A}}} B$ or simply $A \bowtie B$.

Remark 5.5. The analogue of Proposition 4.7 for a matched pair of prealternative algebras contains 20 equations. We omit them. Note that $\left(B, L_{\succ_{A}}, R_{\succ_{A}}, L_{\succ_{A}}, R_{\succ_{A}}\right)$ and $\left(A, L_{\prec_{B}}, R_{\prec_{B}}, L_{\succ_{B}}, R_{\succ_{B}}\right.$ ) must be bimodules of $A$ and $B$, respectively.

Corollary 5.6. Suppose $\left(A, B, L_{\prec_{A}}, R_{\succ_{A}}, L_{\succ_{A}}, R_{\succ_{A}}, L_{\succ_{B}}, R_{\succ_{B}}, L_{\succ_{B}}, R_{\succ_{B}}\right)$ is a matched pair of prealternative algebras. Then

$$
\left(\operatorname{Alt}(A), \operatorname{Alt}(B), L_{\prec_{A}}+L_{\succ_{A}}, R_{\prec_{A}}+R_{\succ_{A}}, L_{\prec_{B}}+L_{\succ_{B}}, R_{\prec_{B}}+R_{\succ_{B}}\right)
$$

is a matched pair of alternative algebras.

Proof. It follows from the relationship between a prealternative algebra and the associated alternative algebra.

Proposition 5.7. Let $\left(A, \prec_{1}, \succ_{1}\right)$ be a prealternative algebra and $\left(\operatorname{Alt}(A), \circ_{1}\right)$ be the associated alternative algebra. Suppose there is a prealternative algebra structure $\prec_{2}, \succ_{2}$ on the dual space $A^{*}$ and $\left(\operatorname{Alt}\left(A^{*}\right), \circ_{2}\right)$ is the associated alternative algebra. Then $\left(\operatorname{Alt}(A), \operatorname{Alt}\left(A^{*}\right), \mathfrak{r}_{<_{1}}^{*}, \mathfrak{l}_{\succ_{1}}^{*}, \mathfrak{r}_{<_{2}}^{*}, \mathfrak{l}_{\succ_{2}}^{*}\right)$ is a matched pair of alternative algebras if and only if $\left(A, A^{*},-\mathfrak{r}_{\succ_{1}}^{*}, \mathfrak{l}_{\mathrm{o}_{1}}^{*}, \mathfrak{r}_{\mathrm{O}_{1}}^{*},-\mathfrak{l}_{<_{1}}^{*},-\mathfrak{r}_{\succ_{2}}^{*}, \mathfrak{l}_{\mathrm{o}_{2}}^{*}, \mathfrak{r}_{\mathrm{o}_{2}}^{*},-\mathfrak{l}_{<_{2}}^{*}\right)$ is also.

Proof. By Corollary 5.6, we only need to prove the "only if" part of the conclusion. If $\left(\operatorname{Alt}(A), \operatorname{Alt}\left(A^{*}\right), \mathfrak{r}_{<_{1}}^{*}, l_{\succ_{1}}^{*}, \mathfrak{r}_{<_{2}}^{*}, \mathfrak{l}_{\succ_{2}}^{*}\right)$ is a matched pair of alternative algebras, then by Proposition 4.8 ,

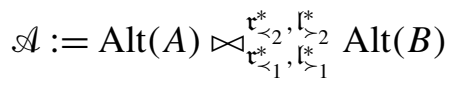

is an $L$-symplectic alternative algebra with symplectic form given by (3-10). Hence Proposition 2.16 gives a compatible prealternative algebra structure on $\mathscr{A}$. Then 
for any $x, y \in A$ and $a^{*}, b^{*} \in A^{*}$ we have

$$
\begin{aligned}
\left\langle a^{*} \prec x, y\right\rangle & =\omega_{p}\left(a^{*} \prec x, y\right)=\omega_{p}\left(a^{*}, x \circ_{1} y\right)=\left\langle\mathfrak{l}_{\iota_{1}}^{*}(x) a^{*}, y\right\rangle, \\
\left\langle a^{*} \prec x, b^{*}\right\rangle & =-\omega_{p}\left(a^{*} \prec x, b^{*}\right) \\
& =-\left\langle a^{*}, \mathfrak{r}_{\succ_{2}}^{*}\left(b^{*}\right) x\right\rangle=-\left\langle b^{*} \succ_{2} a^{*}, x\right\rangle=\left\langle-\mathfrak{r}_{\succ_{2}}^{*}\left(a^{*}\right) x, b^{*}\right\rangle .
\end{aligned}
$$

So $a^{*} \prec x=-\mathfrak{r}_{\succ_{2}}^{*}\left(a^{*}\right) x+\mathfrak{l}_{\circ_{1}}(x) a^{*}$. Similarly,

$$
\begin{aligned}
& x \prec a^{*}=-\mathfrak{r}_{\succ_{1}}^{*}(x) a^{*}+\mathfrak{l}_{\mathrm{o}_{2}}\left(a^{*}\right) x, \quad x \succ a^{*}=\mathfrak{r}_{\mathrm{o}_{1}}^{*}(x) a^{*}-\mathfrak{l}_{\succ_{2}}^{*}\left(a^{*}\right) x, \\
& a^{*} \succ x=\mathfrak{r}_{\mathrm{o}_{2}}\left(a^{*}\right) x-\mathfrak{l}_{\succ_{1}}^{*}(x) a^{*} .
\end{aligned}
$$

Therefore $\left(A, A^{*},-\mathfrak{r}_{\succ_{1}}^{*}, \mathfrak{l}_{\circ_{1}}^{*}, \mathfrak{r}_{\circ_{1}}^{*},-\mathfrak{l}_{<_{1}}^{*},-\mathfrak{r}_{\succ_{2}}^{*}, \mathfrak{l}_{\mathrm{o}_{2}}^{*}, \mathfrak{r}_{\mathrm{o}_{2}}^{*},-\mathfrak{l}_{<_{2}}^{*}\right)$ is a matched pair of prealternative algebras.

\section{Prealternative bialgebras}

Theorem 6.1. Let $(A, \prec, \succ, \alpha, \beta)$ be a prealternative algebra $(A, \prec, \succ)$ equipped with two comultiplications $\alpha, \beta: A \rightarrow A \otimes A$ and let $(\operatorname{Alt}(A), \circ)$ be the associated alternative algebra. Suppose $\alpha^{*}, \beta^{*}: A^{*} \otimes A^{*} \rightarrow A^{*}$ induce a prealternative algebra structure $\prec_{*}, \succ_{*}$ on the dual space $A^{*}$. Then $\left(\operatorname{Alt}(A), \operatorname{Alt}\left(A^{*}\right), \mathfrak{r}_{<}^{*}, \mathfrak{l}_{\succ}^{*}, \mathfrak{r}_{<_{*}^{*}}^{*}, \mathfrak{l}_{\succ_{*}}^{*}\right)$ is a matched pair of alternative algebras if and only if $\alpha, \beta$ satisfy the following eight equations for any $x, y \in A$ :

(6-1) $\alpha(x \circ y+y \circ x)$

$$
=\left(\mathfrak{r}_{\circ}(y) \otimes 1+1 \otimes \mathfrak{l}_{\succ}(y)\right) \alpha(x)+\left(\mathfrak{r}_{\circ}(x) \otimes 1+1 \otimes \mathfrak{l}_{\succ}(x)\right) \alpha(y),
$$

(6-2) $\quad \beta(x \circ y+y \circ x)$

$$
=\left(\mathfrak{r}_{\prec}(y) \otimes 1+1 \otimes \mathfrak{l}_{\circ}(y)\right) \beta(x)+\left(\mathfrak{r}_{\prec}(x) \otimes 1+1 \otimes \mathfrak{l}_{\circ}(x)\right) \beta(y),
$$

(6-3) $\quad \alpha(x \circ y)$

$$
=\left(1 \otimes \mathfrak{r}_{\prec}(x)+1 \otimes \mathfrak{l}_{\succ}(x)-\mathfrak{l}_{\circ}(x) \otimes 1\right) \alpha(y)+\left(\mathfrak{r}_{\circ}(y) \otimes 1\right) \alpha(x)
$$

$$
+\left(\mathfrak{r}_{\circ}(y) \otimes 1-1 \otimes \mathfrak{l}_{\succ}(y)\right) \sigma \beta(x),
$$

(6-4) $\quad \beta(x \circ y)$

$$
\begin{aligned}
=\left(\mathfrak{l}_{\succ}(y) \otimes 1+\mathfrak{r}_{\prec}(y) \otimes 1-1 \otimes \mathfrak{r}_{\circ}(y)\right) \beta(x) & +\left(1 \otimes \mathfrak{l}_{\circ}(x)\right) \beta(y) \\
+ & \left(1 \otimes \mathfrak{l}_{\circ}(x)-\mathfrak{r}_{\prec}(x) \otimes 1\right) \sigma \alpha(y),
\end{aligned}
$$

(6-5) $\quad(\alpha+\beta)(x \prec y)$

$$
\begin{aligned}
= & \left(1 \otimes \mathfrak{l}_{\prec}(x)\right)(\sigma \alpha+\beta)(y) \\
& +\left(\mathfrak{r}_{\prec}(y) \otimes 1+\mathfrak{l}_{\succ}(y) \otimes 1-1 \otimes \mathfrak{r}_{\prec}(y)\right)(\alpha+\beta)(x)
\end{aligned}
$$


$(6-6) \quad(\alpha+\beta)(x \succ y)$

$$
\begin{aligned}
= & \left(\mathfrak{r}_{\succ}(y) \otimes 1\right)(\alpha+\sigma \beta)(x) \\
& +\left(1 \otimes \mathfrak{l}_{\succ}(x)+1 \otimes \mathfrak{r}_{\prec}(x)-\mathfrak{l}_{\succ}(x) \otimes 1\right)(\alpha+\beta)(y)
\end{aligned}
$$

$-\left(1 \otimes \mathfrak{l}_{\prec}(y)\right) \sigma \alpha(x)$,

(6-7) $\quad(\alpha+\beta+\sigma \alpha+\sigma \beta)(x \succ y)$

$$
=\left(\mathfrak{r}_{\succ}(y) \otimes 1\right) \alpha(x)+\left(1 \otimes \mathfrak{l}_{\succ}(x)\right)(\alpha+\beta)(y)+\left(1 \otimes \mathfrak{r}_{\succ}(y)\right) \sigma \alpha(x)
$$$$
+\left(\mathfrak{l}_{\succ}(x) \otimes 1\right)(\sigma \alpha+\sigma \beta)(y),
$$

(6-8) $\quad(\alpha+\beta+\sigma \alpha+\sigma \beta)(x \prec y)$

$$
\begin{aligned}
=\left(1 \otimes \mathfrak{l}_{\prec}(x)\right) \beta(y)+\left(\mathfrak{r}_{\prec}(y) \otimes 1\right)(\alpha+\beta)(x) & +\left(\mathfrak{l}_{\prec}(x) \otimes 1\right) \sigma \beta(y) \\
& +\left(1 \otimes \mathfrak{r}_{\prec}(y)\right)(\sigma \alpha+\sigma \beta)(x) .
\end{aligned}
$$

Proof. By Proposition 4.7, we need to prove (6-1)-(6-8) are equivalent to (4-3)(4-9) if we replace $\left(A, B, L_{A}, R_{A}, L_{B}, R_{B}\right)$ by $\left(\operatorname{Alt}(A), \operatorname{Alt}\left(A^{*}\right), \mathfrak{r}_{<}^{*}, \mathfrak{l}_{\succ}^{*}, \mathfrak{r}_{\succ_{*}}^{*}, \mathfrak{l}_{\succ_{*}}^{*}\right)$. As an example, we give an explicit proof of the equivalence between (4-3) and (6-3). The proofs of the others are similar. In this case, (4-3) becomes

$$
\begin{aligned}
\mathfrak{r}_{<}^{*}\left(\mathfrak{r}_{<}^{*}(x) a^{*}+\mathfrak{l}_{\succ}^{*}(x) a^{*}\right) y+\left(\mathfrak{r}_{<}^{*}\left(a^{*}\right) x+\mathfrak{l}_{\succ}^{*}\left(a^{*}\right) x\right) \circ y \\
\quad=\mathfrak{r}_{<}^{*}\left(a^{*}\right)(x \circ y)+\mathfrak{l}_{\succ}^{*}\left(\mathfrak{l}_{\succ}^{*}(y) a^{*}\right) x+x \circ\left(\mathfrak{r}_{<}^{*}\left(a^{*}\right) y\right),
\end{aligned}
$$

where $x, y \in A$ and $a^{*} \in A^{*}$. Let both the left and the right side of this equation act on $b^{*} \in A^{*}$. Then

$$
\begin{aligned}
\left\langle\text { l.h.s., } b^{*}\right\rangle= & \left\langle\mathfrak{r}_{\prec}^{*}\left(\mathfrak{r}_{\prec}^{*}(x) a^{*}+\mathfrak{l}_{\succ}^{*}(x) a^{*}\right) y+\left(\mathfrak{r}_{\prec}^{*}\left(a^{*}\right) x+\mathfrak{l}_{\succ}^{*}\left(a^{*}\right) x\right) \circ y, b^{*}\right\rangle \\
= & \left\langle y, b^{*} \prec\left(\mathfrak{r}_{\prec}^{*}(x) a^{*}+\mathfrak{l}_{\succ}^{*}(x) a^{*}\right)\right\rangle+\left\langle\mathfrak{r}_{\prec}^{*}\left(a^{*}\right) x+\mathfrak{l}_{\succ}^{*}\left(a^{*}\right) x, \mathfrak{r}_{\circ}^{*}(y) b^{*}\right\rangle \\
= & \left\langle\alpha(y), b^{*} \otimes \mathfrak{r}_{\prec}^{*}(x) a^{*}+b^{*} \otimes \mathfrak{l}_{\succ}^{*}(x) a^{*}\right\rangle+\left\langle\alpha(x), \mathfrak{r}_{\circ}^{*}(y) b^{*} \otimes a^{*}\right\rangle \\
& +\left\langle\beta(x), a^{*} \otimes \mathfrak{r}_{\circ}^{*}(y) b^{*}\right\rangle \\
= & \left\langle\left(1 \otimes \mathfrak{r}_{\prec}(x)+1 \otimes \mathfrak{l}_{\succ}(x)\right) \alpha(y)+\left(\mathfrak{r}_{\circ}(y) \otimes 1\right)(\alpha+\sigma \beta)(x), b^{*} \otimes a^{*}\right\rangle, \\
\left\langle\text { r.h.s., } b^{*}\right\rangle= & \left\langle x \circ y, b^{*} \prec a^{*}\right\rangle+\left\langle x,\left(\mathfrak{l}_{\succ}^{*}(y) a^{*}\right) \succ b^{*}\right\rangle+\left\langle\mathfrak{r}_{\prec}^{*}\left(a^{*}\right) y, \mathfrak{l}_{\circ}^{*}(x) b^{*}\right\rangle \\
= & \left\langle\alpha(x \circ y), b^{*} \otimes a^{*}\right\rangle+\left\langle\beta(x), \mathfrak{l}_{\succ}^{*}(y) a^{*} \otimes b^{*}\right\rangle+\left\langle\alpha(y), \mathfrak{l}_{\circ}^{*}(x) b^{*} \otimes a^{*}\right\rangle \\
= & \left\langle\alpha(x \circ y)+\left(1 \otimes \mathfrak{l}_{\succ}(y)\right) \sigma \beta(x)+\left(\mathfrak{l}_{\circ}(x) \otimes 1\right) \alpha(y), b^{*} \otimes a^{*}\right\rangle .
\end{aligned}
$$

So (4-3) holds if and only if (6-3) holds.

Definition 6.2. (1) Let $(A, \alpha, \beta)$ be a vector space with two comultiplications $\alpha, \beta: A \rightarrow A \otimes A$. If $\left(A, \alpha^{*}, \beta^{*}\right)$ is a prealternative algebra, then we call the triple $(A, \alpha, \beta)$ a prealternative coalgebra.

(2) If $(A, \prec, \succ, \alpha, \beta)$ is a prealternative algebra $(A, \prec, \succ)$ with two comultiplications $\alpha, \beta: A \rightarrow A \otimes A$ such that $(A, \alpha, \beta)$ is a prealternative coalgebra 
and $\alpha$ and $\beta$ satisfy (6-1)-(6-8), then we call $(A, \prec, \succ, \alpha, \beta)$ a prealternative bialgebra.

Combining Propositions 4.8, 5.7 and Theorem 6.1, we have this:

Corollary 6.3. Let $\left(A, \prec_{1}, \succ_{1}\right)$ be a prealternative algebra and $\left(\operatorname{Alt}(A), \circ_{1}\right)$ be the associated alternative algebra. Let $\alpha, \beta: A \rightarrow A \otimes A$ be two linear maps such that $\alpha^{*}, \beta^{*}: A^{*} \otimes A^{*} \subset(A \otimes A)^{*} \rightarrow A^{*}$ induce a prealternative algebra structure $\prec_{2}, \succ_{2}$ on $A^{*}$, that is, $(A, \alpha, \beta)$ is a prealternative coalgebra. Let $\left(\operatorname{Alt}\left(A^{*}\right), \circ_{2}\right)$ be the associated alternative algebra of $\left(A^{*}, \prec_{2}, \succ_{2}\right)$. Then the following conditions are equivalent:

(1) $\left(\operatorname{Alt}(A) \bowtie \operatorname{Alt}\left(A^{*}\right), \operatorname{Alt}(A), \operatorname{Alt}\left(A^{*}\right), \omega_{p}\right)$ is an L-symplectic alternative algebra (or a phase space of $\operatorname{Alt}(A))$, where $\omega_{p}$ is given by (3-10).

(2) $\left(\operatorname{Alt}(A), \operatorname{Alt}\left(A^{*}\right), \mathfrak{r}_{\succ_{1}}^{*}, \mathfrak{l}_{\succ_{1}}^{*}, \mathfrak{r}_{<_{2}}^{*}, \mathfrak{l}_{\succ_{2}}^{*}\right)$ is a matched pair of alternative algebras.

(3) $\left(A, A^{*},-\mathfrak{r}_{\succ_{1}}^{*}, \mathfrak{r}_{\mathrm{o}_{1}}^{*}, \mathfrak{r}_{\mathrm{o}_{1}}^{*},-\mathfrak{l}_{<_{1}}^{*},-\mathfrak{r}_{\succ_{2}}^{*}, \mathfrak{l}_{\mathrm{o}_{2}}^{*}, \mathfrak{r}_{\mathrm{o}_{2}}^{*},-\mathfrak{l}_{<_{2}}^{*}\right)$ is a matched pair of prealternative algebras.

(4) $\left(A, \prec_{1}, \succ_{1}, \alpha, \beta\right)$ is a prealternative bialgebra.

Definition 6.4. Let $\left(A, \prec_{A}, \succ_{A}, \alpha_{A}, \beta_{A}\right)$ and $\left(B, \prec_{B}, \succ_{B}, \alpha_{B}, \beta_{B}\right)$ be two prealternative bialgebras. A homomorphism of prealternative bialgebras $\varphi: A \rightarrow B$ is a homomorphism of prealternative algebras such that

(6-9) $(\varphi \otimes \varphi) \alpha_{A}(x)=\alpha_{B}(\varphi(x)) \quad$ and $\quad(\varphi \otimes \varphi) \beta_{A}(x)=\beta_{B}(\varphi(x)) \quad$ for all $x \in A$.

Proposition 6.5. Two L-symplectic (hence phase spaces of) alternative algebras are isomorphic if and only if their corresponding prealternative bialgebras are isomorphic.

Proof. Let $\left(\operatorname{Alt}(C) \bowtie \operatorname{Alt}\left(C^{*}\right), \operatorname{Alt}(C), \operatorname{Alt}\left(C^{*}\right), \omega_{p}\right)$ for $C=A, B$ be two $L$ symplectic alternative algebras, with $\varphi: \operatorname{Alt}(A) \bowtie \operatorname{Alt}\left(A^{*}\right) \rightarrow \operatorname{Alt}(B) \bowtie \operatorname{Alt}\left(B^{*}\right)$ the isomorphism. Then $\left.\varphi\right|_{A}: A \rightarrow B$ and $\left.\varphi\right|_{A^{*}}: A^{*} \rightarrow B^{*}$ are isomorphisms of prealternative algebras by Proposition 4.2. Moreover, $\left.\varphi\right|_{A^{*}}=\left(\left.\varphi\right|_{A}\right)^{*-1}$ since

$$
\begin{aligned}
\left\langle\left.\varphi\right|_{A^{*}}\left(a^{*}\right), \varphi(x)\right\rangle & =\omega_{p}\left(\left.\varphi\right|_{A^{*}}\left(a^{*}\right), \varphi(x)\right)=\omega_{p}\left(a^{*}, x\right)=\left\langle a^{*}, x\right\rangle \\
& =\left\langle\varphi^{*}\left(\left.\varphi\right|_{A}\right)^{*-1}\left(a^{*}\right), x\right\rangle \\
& =\left\langle\left(\left.\varphi\right|_{A}\right)^{*-1}\left(a^{*}\right), \varphi(x)\right\rangle \text { for all } x \in A \text { and } a^{*} \in A^{*} .
\end{aligned}
$$

So $\left(\left.\varphi\right|_{A}\right)^{*}: B^{*} \rightarrow A^{*}$ is a homomorphism of prealternative algebras, and then $\left(A, \prec_{A}, \succ_{A}, \alpha_{A}, \beta_{A}\right)$ and $\left(B, \prec_{B}, \succ_{B}, \alpha_{B}, \beta_{B}\right)$ are isomorphic as prealternative bialgebras. Conversely, suppose these two are isomorphic prealternative bialgebras, and let $\varphi^{\prime}: A \rightarrow B$ be the isomorphism. Let $\varphi: A \oplus A^{*} \rightarrow B \oplus B^{*}$ be a linear map defined by

$$
\varphi(x)=\varphi^{\prime}(x) \text { and } \varphi\left(a^{*}\right)=\left(\varphi^{*}\right)^{-1}\left(a^{*}\right) \quad \text { for all } x \in A \text { and } a^{*} \in A^{*} .
$$


Then it is easy to show that $\varphi$ is an isomorphism of the two $L$-symplectic alternative algebras in the statement.

Example 6.6. Let $(A, \prec, \succ, \alpha, \beta)$ be a prealternative bialgebra. Then the dual $\left(A, \prec_{*}, \succ_{*}, \gamma, \delta\right)$ is also a prealternative bialgebra, where the prealternative algebra structure $\prec, \succ$ on $A$ is defined by the linear maps $\gamma^{*}, \delta^{*}: A \otimes A \rightarrow A$, and $\alpha^{*}, \beta^{*}: A^{*} \otimes A^{*} \rightarrow A^{*}$ induce a prealternative algebra structure $\prec_{*}, \succ_{*}$ on $A^{*}$.

Example 6.7. Let $(A, \prec, \succ)$ be a prealternative algebra. Then $(A, \prec, \succ, 0,0)$ is a prealternative bialgebra, and the corresponding prealternative algebra structure on $A \oplus A^{*}$ is the semidirect sum $A \ltimes_{-\mathfrak{r}_{\curvearrowright}^{*},,_{0}^{*}, \mathfrak{r}_{\circ}^{*},-\mathfrak{r}_{<}^{*}} A^{*}$. The corresponding associated alternative algebra is the semidirect $\operatorname{sum} \operatorname{Alt}(A) \ltimes_{\mathfrak{r}_{<}^{*}, l_{<}^{*}} A^{*}$, with symplectic form $\omega_{p}$ given by (3-10).

\section{Coboundary prealternative bialgebras}

Definition 7.1. A prealternative bialgebra $(A, \prec, \succ, \alpha, \beta)$ is called coboundary if the linear maps $\alpha, \beta: A \rightarrow A \otimes A$ are given by

$$
\begin{aligned}
& \alpha(x)=\left(\mathfrak{r}_{\circ}(x) \otimes 1-1 \otimes \mathfrak{l}_{\succ}(x)\right) r_{\prec}, \\
& \beta(x)=\left(1 \otimes \mathfrak{l}_{\circ}(x)-\mathfrak{r}_{\prec}(x) \otimes 1\right) r_{\succ},
\end{aligned}
$$

where $x \circ y=x \prec y+x \succ y, x, y \in A$ and $r_{\prec}, r_{\succ} \in A \otimes A$.

Remark 7.2. The expression of (7-1) and (6-1)-(6-2) looks like certain kind of 1 -coboundary and 1-cocycle.

Theorem 7.3. Let $(A, \prec, \succ)$ be a prealternative algebra with two linear maps $\alpha, \beta: A \rightarrow A \otimes A$ defined by (7-1). If $r_{\prec}=r_{\succ}=r \in A \otimes A$ and $r$ is symmetric, then $\alpha, \beta$ satisfy (6-1)-(6-8).

Proof. It is obvious that $\alpha, \beta$ automatically satisfy (6-1) and (6-2). For (6-3)-(6-8), we give as an example an explicit proof of the fact that $\alpha, \beta$ satisfy (6-5); the proof of the other cases is similar. Assume $r=\sum_{i} u_{i} \otimes v_{i} \in A \otimes A$. After rearranging the terms suitably, we have, noting that $r$ is symmetric,

$$
\begin{aligned}
& (\alpha+\beta)(x \prec y)-\left(1 \otimes \mathfrak{l}_{<}(x)\right)(\sigma \alpha+\beta)(y) \\
& -\left(\mathfrak{r}_{\prec}(y) \otimes 1+\mathfrak{l}_{\succ}(y) \otimes 1-1 \otimes \mathfrak{r}_{\prec}(y)\right)(\alpha+\beta)(x)+\left(\mathfrak{r}_{\succ}(x) \otimes 1\right) \sigma \beta(y) \\
& =\sum_{i}\left(u_{i} \circ(x \prec y) \otimes v_{i}-u_{i} \prec(x \prec y) \otimes v_{i}-\left(u_{i} \circ x\right) \prec y \otimes v_{i}+\left(u_{i} \prec x\right) \prec y \otimes v_{i}\right. \\
& -y \succ\left(u_{i} \circ x\right) \otimes v_{i}+y \succ\left(u_{i} \prec x\right) \otimes v_{i}+\left(y \circ u_{i}\right) \succ x \otimes v_{i}-u_{i} \otimes(x \prec y) \succ v_{i} \\
& +u_{i} \otimes(x \prec y) \circ v_{i}-u_{i} \otimes x \prec\left(v_{i} \circ y\right)-u_{i} \otimes x \prec\left(y \circ v_{i}\right)-u_{i} \otimes\left(x \succ v_{i}\right) \prec y \\
& +u_{i} \otimes\left(x \circ v_{i}\right) \prec y+y \succ u_{i} \otimes x \prec v_{i}+y \succ u_{i} \otimes x \succ v_{i}-y \succ u_{i} \otimes x \circ v_{i}+u_{i} \prec y \otimes x \prec v_{i} \\
& \left.+u_{i} \prec y \otimes x \succ v_{i}-u_{i} \prec y \otimes x \circ v_{i}+u_{i} \circ x \otimes v_{i} \prec y-u_{i} \prec x \otimes v_{i} \prec y-u_{i} \succ x \otimes v_{i} \prec y\right) \text {. }
\end{aligned}
$$


The sum of the first seven terms is zero since it is equal to

$$
\sum_{i}\left(u_{i} \succ(x \prec y)-\left(u_{i} \succ x\right) \prec y-y \succ\left(u_{i} \succ x\right)+\left(y \circ u_{i}\right) \succ x\right) \otimes v_{i}=0 .
$$

The sum of the 8th through the 13th term is zero since it is equal to

$$
\sum_{i} u_{i} \otimes\left((x \prec y) \prec v_{i}-x \prec\left(y \circ v_{i}\right)-x \prec\left(v_{i} \circ y\right)+\left(x \prec v_{i}\right) \prec y\right)=0 .
$$

The sum of the 14th through 16th term, the sum of 17th through 19th term, and the sum of the last three terms are all zero obviously.

Lemma 7.4. Let $A$ be a vector space and $\alpha, \beta: A \rightarrow A \otimes A$ be two linear maps. Then $(A, \alpha, \beta)$ is a prealternative coalgebra if and only if the linear maps $S_{\alpha, \beta}^{i}$ : $A \rightarrow A \otimes A \otimes A$ for $i=1$, 2, 3, 4 given by the following equations are all zero for any $x \in A$ :

$$
\begin{gathered}
S_{\alpha, \beta}^{1}(x)=((\alpha+\beta) \otimes 1) \beta(x)+(\sigma \otimes 1)((\alpha+\beta) \otimes 1) \beta(x) \\
-(1 \otimes \beta) \beta(x)-(\sigma \otimes 1)(1 \otimes \beta) \beta(x), \\
S_{\alpha, \beta}^{2}(x)=(\beta \otimes 1) \alpha(x)+(\sigma \otimes 1)(\alpha \otimes 1) \alpha(x)
\end{gathered}
$$

$$
-(1 \otimes \alpha) \beta(x)-(\sigma \otimes 1)(1 \otimes(\alpha+\beta)) \alpha(x),
$$

$$
\begin{aligned}
& S_{\alpha, \beta}^{3}(x)=((\alpha+\beta) \otimes 1) \beta(x)+(1 \otimes \sigma)(\beta \otimes 1) \alpha(x) \\
& -(1 \otimes \beta) \beta(x)-(1 \otimes \sigma)(1 \otimes \alpha) \beta(x), \\
& S_{\alpha, \beta}^{4}(x)=(\alpha \otimes 1) \alpha(x)+(1 \otimes \sigma)(\alpha \otimes 1) \alpha(x) \\
& -(1 \otimes(\alpha+\beta)) \alpha(x)-(1 \otimes \sigma)(1 \otimes(\alpha+\beta)) \alpha(x) .
\end{aligned}
$$

Proof. It follows immediately from the definition 2.6 of a prealternative algebra.

Definition 7.5. Let $(A, \prec, \succ)$ be a prealternative algebra and $(\operatorname{Alt}(A), \circ)$ be the associated alternative algebra. Let $r \in A \otimes A$. The following equations are called $\mathrm{PA}_{j}^{i}$ equations for $i=1,2$ and $j=1,2,3$ :

$$
\begin{aligned}
& \mathrm{PA}_{1}^{1}=r_{12} \circ r_{13}-r_{23} \succ r_{12}-r_{13} \prec r_{23}=0, \\
& \mathrm{PA}_{1}^{2}=r_{13} \circ r_{12}-r_{12} \prec r_{23}-r_{23} \succ r_{13}=0, \\
& \mathrm{PA}_{2}^{1}=r_{12} \circ r_{23}-r_{23} \prec r_{13}-r_{13} \succ r_{12}=0, \\
& \mathrm{PA}_{2}^{2}=r_{23} \circ r_{12}-r_{13} \succ r_{23}-r_{12} \prec r_{13}=0, \\
& \mathrm{PA}_{3}^{1}=r_{13} \circ r_{23}-r_{12} \succ r_{13}-r_{23} \prec r_{12}=0, \\
& \mathrm{PA}_{3}^{2}=r_{23} \circ r_{13}-r_{13} \prec r_{12}-r_{12} \succ r_{23}=0 .
\end{aligned}
$$

We set $\mathrm{PA}_{j}=\mathrm{PA}_{j}^{1}+\mathrm{PA}_{j}^{2}$, where $j=1,2,3$. Collectively the $\mathrm{PA}_{j}^{i}$ equations are called the PA equations. 
Proposition 7.6. Let $(A, \prec, \succ)$ be a prealternative algebra and $(\operatorname{Alt}(A), \circ)$ be the associated alternative algebra. Let $r \in A \otimes A$ be symmetric. Let $\alpha, \beta: A \rightarrow A \otimes A$ be two linear maps given by (7-1), where $r_{\prec}=r_{\succ}=r$. Then $(A, \alpha, \beta)$ becomes $a$ prealternative coalgebra if and only if for any $x \in A$

$-\left(1 \otimes 1 \otimes \mathfrak{l}_{\circ}(x)\right) \mathrm{PA}_{3}+\left(1 \otimes \mathfrak{r}_{\prec}(x) \otimes 1\right) \mathrm{PA}_{3}^{2}+\left(\mathfrak{r}_{\prec}(x) \otimes 1 \otimes 1\right) \mathrm{PA}_{3}^{1}=0$, $-\left(1 \otimes 1 \otimes \mathfrak{l}_{\succ}(x)\right) \mathrm{PA}_{2}+\left(1 \otimes \mathfrak{r}_{\circ}(x) \otimes 1\right) \mathrm{PA}_{2}^{1}+\left(\mathfrak{r}_{\prec}(x) \otimes 1 \otimes 1\right) \mathrm{PA}_{2}^{2}=0$, $\left(\mathfrak{r}_{\prec}(x) \otimes 1 \otimes 1\right) \mathrm{PA}_{3}-\left(1 \otimes 1 \otimes \mathfrak{l}_{\circ}(x)\right) \mathrm{PA}_{3}^{1}-\left(1 \otimes \mathfrak{l}_{\succ}(x) \otimes 1\right) \mathrm{PA}_{3}^{2}=0$, $\left(\mathfrak{r}_{\circ}(x) \otimes 1 \otimes 1\right) \mathrm{PA}_{1}-\left(1 \otimes \mathfrak{l}_{\succ}(x) \otimes 1\right) \mathrm{PA}_{1}^{2}-\left(1 \otimes 1 \otimes \mathfrak{l}_{\succ}(x)\right) \mathrm{PA}_{1}^{1}=0$.

Proof. We give an explicit proof of the fact that the first of (7-4) is equivalent to $S_{\alpha, \beta}^{1}=0$ as an example. Using a similar argument, we can show that the rest are respectively equivalent to $S_{\alpha, \beta}^{i}=0$ for $i=2,3,4$. Set $r=\sum_{i} u_{i} \otimes v_{i}$. Substituting $\alpha(x)=\sum_{i} u_{i} \circ x \otimes v_{i}-u_{i} \otimes x \succ v_{i} \quad$ and $\quad \beta(x)=\sum_{i} u_{i} \otimes x \circ v_{i}-u_{i} \prec x \otimes v_{i}$

for all $x \in A$ into the first of (7-2) and after rearranging the terms suitably, we divide $S_{\alpha, \beta}^{1}$ as

$$
S_{\alpha, \beta}^{1}=(\mathrm{S} 1)+(\mathrm{S} 2)+(\mathrm{S} 3),
$$

where

$$
\begin{aligned}
& (\mathrm{S} 1)=\sum_{i, j}\left(u_{i} \circ u_{j} \otimes v_{i} \otimes x \circ v_{j}-u_{i} \otimes u_{j} \succ v_{i} \otimes x \circ v_{j}+u_{i} \otimes u_{j} \circ v_{i} \otimes x \circ v_{j}\right. \\
& -u_{i} \prec u_{j} \otimes v_{i} \otimes x \circ v_{j}+v_{i} \otimes u_{i} \circ u_{j} \otimes x \circ v_{j}-u_{j} \succ v_{i} \otimes u_{i} \otimes x \circ v_{j} \\
& +u_{j} \circ v_{i} \otimes u_{i} \otimes x \circ v_{j}-v_{i} \otimes u_{i} \prec u_{j} \otimes x \circ v_{j}-u_{j} \otimes u_{i} \otimes\left(x \circ v_{j}\right) \circ v_{i} \\
& \left.-u_{i} \otimes u_{j} \otimes\left(x \circ v_{j}\right) \circ v_{i}\right) \text {. } \\
& \begin{array}{r}
(\mathrm{S} 2)=\sum_{i, j}\left(u_{i} \otimes\left(u_{j} \prec x\right) \succ v_{i} \otimes v_{j}-u_{i} \otimes\left(u_{j} \prec x\right) \circ v_{i} \otimes v_{j}\right. \\
-v_{i} \otimes u_{i} \circ\left(u_{j} \prec x\right) \otimes v_{j}+
\end{array} \\
& -v_{i} \otimes u_{i} \circ\left(u_{j} \prec x\right) \otimes v_{j}+v_{i} \otimes u_{i} \prec\left(u_{j} \prec x\right) \otimes v_{j} \\
& \left.+u_{j} \otimes u_{i} \prec\left(x \circ v_{j}\right) \otimes v_{i}+u_{i} \otimes u_{j} \prec x \otimes v_{j} \circ v_{i}-u_{i} \prec v_{j} \otimes u_{j} \prec x \otimes v_{i}\right), \\
& (\mathrm{S} 3)=\sum_{i, j}\left(-u_{i} \circ\left(u_{j} \prec x\right) \otimes v_{i} \otimes v_{j}+u_{i} \prec\left(u_{j} \prec x\right) \otimes v_{i} \otimes v_{j}\right. \\
& +\left(u_{j} \prec x\right) \succ v_{i} \otimes u_{i} \otimes v_{j}-\left(u_{j} \prec x\right) \circ v_{i} \otimes u_{i} \otimes v_{j}+u_{j} \prec x \otimes u_{i} \otimes v_{j} \circ v_{i} \\
& \left.-u_{j} \prec x \otimes u_{i} \prec v_{j} \otimes v_{i}+u_{i} \prec\left(x \circ v_{j}\right) \otimes u_{j} \otimes v_{i}\right) .
\end{aligned}
$$

Since $r$ is symmetric and by Remark 2.6, we have

$$
\begin{aligned}
& (\mathrm{S} 1)=-\left(1 \otimes 1 \otimes \mathfrak{l}_{\circ}(x)\right) \mathrm{PA}_{3}, \quad(\mathrm{~S} 2)=\left(1 \otimes \mathfrak{r}_{\prec}(x) \otimes 1\right) \mathrm{PA}_{3}^{2}, \\
& (\mathrm{~S} 3)=\left(\mathfrak{r}_{\prec}(x) \otimes 1 \otimes 1\right) \mathrm{PA}_{3}^{1} .
\end{aligned}
$$


Theorem 7.7. Let $(A, \prec, \succ)$ be a prealternative algebra and $r \in A \otimes A$ be symmetric. Let $\alpha, \beta: A \rightarrow A \otimes A$ be linear maps given by (7-1), where $r_{\prec}=r_{\succ}=r$. Then $(A, \prec, \succ, \alpha, \beta)$ is a prealternative bialgebra if and only if the equations of (7-4) are satsified.

Proof. It follows from Theorem 7.3 and Proposition 7.6.

Next we give a Drinfeld double construction [Chari and Pressley 1994] for a prealternative bialgebra.

Theorem 7.8. Let $(A, \prec, \succ, \alpha, \beta)$ be a prealternative bialgebra. Then there is a canonical prealternative bialgebra structure on $A \oplus A^{*}$ such that the inclusions $i_{1}: A \rightarrow A \oplus A^{*}$ and $i_{2}: A^{*} \rightarrow A \oplus A^{*}$ into the two summands are homomorphisms of prealternative bialgebras, where the prealternative bialgebra structure on $A^{*}$ is given in Example 6.6.

Proof. Denote the prealternative algebra structures on $A^{*}$ induced by $\alpha^{*}$ and $\beta^{*}$ by $\prec_{*}$ and $\succ_{*}$ respectively, and the associated alternative algebra structure by $*$. Let $r \in A \otimes A^{*} \subset\left(A \oplus A^{*}\right) \otimes\left(A \oplus A^{*}\right)$ correspond to the identity map id : $A \rightarrow A$. Then the prealternative algebra structure $\prec_{\bullet}, \succ_{\bullet}$ on $A \oplus A^{*}$ is given by

$$
\mathscr{P} \mathscr{A D}(A)=A \bowtie_{-\mathfrak{r}_{>}^{*}, \mathfrak{l}_{0}^{*}, \mathfrak{r}_{0}^{*},-\mathfrak{l}_{<}^{*}}^{-\mathfrak{r}_{*}^{*}, r_{*}^{*}, \mathfrak{r}_{*}^{*},-r^{*}} A^{*},
$$

that is, for all $x, y \in A$ and $a^{*}, b^{*} \in A^{*}$,

$$
\begin{aligned}
& x \prec \bullet y=x \prec y, \\
& x \succ \bullet y=x \succ y, \\
& a^{*} \prec_{\bullet} b^{*}=a^{*} \prec_{*} b^{*} \text {, } \\
& a^{*} \succ_{\bullet} b^{*}=a^{*} \succ_{*} b^{*} \text {, } \\
& x \prec \bullet a^{*}=-\mathfrak{r}_{\succ}^{*}(x) b^{*}+l_{*}^{*}\left(a^{*}\right) x, \quad x \succ_{\bullet} a^{*}=\mathfrak{r}_{\circ}^{*}(x) a^{*}-\mathfrak{l}_{\succ_{*}^{*}}\left(a^{*}\right) x, \\
& a^{*} \prec \bullet x=-\mathfrak{r}_{\succ_{*}}^{*}\left(a^{*}\right) x+\mathfrak{l}_{\circ}^{*}(x) a^{*}, \quad a^{*} \succ \bullet x=\mathfrak{r}_{*}^{*}\left(a^{*}\right) x-\mathfrak{l}_{\prec}^{*}(x) a^{*},
\end{aligned}
$$

We denote its associated alternative algebra structure by $\bullet$. Let $\left\{e_{i}, \ldots, e_{n}\right\}$ be a basis of $A$ and $\left\{e_{1}^{*}, \ldots, e_{n}^{*}\right\}$ be the dual basis. Then $r=\sum_{i} e_{i} \otimes e_{i}^{*}$. Next we prove that

$\alpha_{\mathscr{P} \mathscr{A}}(u)=\left(\mathfrak{r}_{\circ}(u) \otimes 1-1 \otimes \mathfrak{l}_{\succ}(u)\right) r \quad$ and $\quad \beta_{\mathscr{P} \mathscr{A}}(u)=\left(1 \otimes \mathfrak{l}_{\circ}(u)-\mathfrak{r}_{<}(u) \otimes 1\right) r$ induce a (coboundary) prealternative bialgebra structure on $A \oplus A^{*}$. Since $r$ is not symmetric we cannot apply Theorem 7.7 and $\beta_{\mathscr{P} \mathscr{A}}$ satisfies (6-1)-(6-8) and the conditions of Lemma 7.4. For the former, we prove that $\alpha_{\mathscr{P} \mathscr{A} \mathscr{D}}$ and $\beta_{\mathscr{P} \mathscr{A} \mathscr{D}}$ satisfy (6-3) as an example; the proof of the others is similar. In fact, we only need to prove

$$
\left(\mathfrak{r}_{\circ}(y) \otimes 1-1 \otimes \mathfrak{l}_{\succ}(y)\right)\left(\mathfrak{l}_{\circ}(x) \otimes 1-1 \otimes \mathfrak{r}_{\prec}(x)\right)(r-\sigma r)=0 \quad \text { for all } x \in A .
$$

We can prove this equation in the following cases: $x, y \in A ; x, y \in A^{*} ; x \in A$ and $y \in A^{*}$; and $x \in A^{*}$ and $y \in A$. We prove the first case; the proof of the others 
is similar. Let $x=e_{i}$ and $y=e_{j}$; then the equation becomes

$$
\sum_{k}\left(\left(e_{i} \bullet e_{k}^{*}\right) \bullet e_{j} \otimes e_{k}-e_{k}^{*} \bullet e_{j} \otimes e_{k} \prec \bullet e_{i}-e_{i} \bullet e_{k}^{*} \otimes e_{j} \succ \bullet e_{k}+e_{k}^{*} \otimes e_{j} \succ \bullet\left(e_{k} \prec \bullet e_{i}\right)\right)
$$

$=\sum_{k}\left(\left(e_{i} \bullet e_{k}\right) \bullet e_{j} \otimes e_{k}^{*}-e_{k} \bullet e_{j} \otimes e_{k}^{*} \prec \bullet e_{i}-e_{i} \bullet e_{k} \otimes e_{j} \succ \bullet e_{k}^{*}+e_{k} \otimes e_{j} \succ \bullet\left(e_{k}^{*} \prec \bullet e_{i}\right)\right)$.

The coefficient of $e_{m} \otimes e_{n}$ on the left side of (7-5) is

$$
\begin{gathered}
\sum_{k}\left(\left\langle\left(e_{i} \bullet e_{n}^{*}\right) \bullet e_{j}, e_{m}^{*}\right\rangle-\left\langle e_{k}^{*} \bullet e_{j}, e_{m}^{*}\right\rangle\left\langle e_{k} \prec \bullet e_{i}, e_{n}^{*}\right\rangle-\left\langle e_{i} \bullet e_{k}^{*}, e_{m}^{*}\right\rangle\left\langle e_{j} \succ \bullet e_{k}, e_{n}^{*}\right\rangle\right) \\
=\sum_{k}\left(\left\langle e_{n}^{*}, e_{k} \prec e_{i}\right\rangle\left\langle e_{j}, e_{m}^{*} \prec_{*} e_{k}^{*}\right\rangle+\left\langle e_{i}, e_{n}^{*} \succ_{*} e_{k}^{*}\right\rangle\left\langle e_{k} \circ e_{j}, e_{m}^{*}\right\rangle\right. \\
\left.-\left\langle e_{j}, e_{m}^{*} \prec_{*} e_{k}^{*}\right\rangle\left\langle e_{k} \prec e_{i}, e_{n}^{*}\right\rangle-\left\langle e_{i}, e_{k}^{*} \succ_{*} e_{m}^{*}\right\rangle\left\langle e_{j} \succ e_{k}, e_{n}^{*}\right\rangle\right),
\end{gathered}
$$

while on the right side that coefficient is the same:

$$
\begin{gathered}
\sum_{k}\left(-\left\langle e_{k} \bullet e_{j}, e_{m}^{*}\right\rangle\left\langle e_{k}^{*} \prec \bullet e_{i}, e_{n}^{*}\right\rangle-\left\langle e_{i} \bullet e_{k}, e_{m}^{*}\right\rangle\left\langle e_{j} \succ_{\bullet} e_{k}^{*}, e_{n}^{*}\right\rangle+\left\langle e_{j} \succ_{\bullet}\left(e_{m}^{*} \prec \bullet e_{i}\right), e_{n}^{*}\right\rangle\right) \\
=\sum_{k}\left(\left\langle e_{k} \circ e_{j}, e_{m}^{*}\right\rangle\left\langle e_{i}, e_{n}^{*} \succ_{*} e_{k}^{*}\right\rangle+\left\langle e_{i} \circ e_{k}, e_{m}^{*}\right\rangle\left\langle e_{j}, e_{k}^{*} \prec_{*} e_{n}^{*}\right\rangle\right. \\
\left.-\left\langle e_{j} \succ e_{k}, e_{n}^{*}\right\rangle\left\langle e_{i}, e_{k}^{*} \succ_{*} e_{m}^{*}\right\rangle-\left\langle e_{j}, e_{k}^{*} \prec_{*} e_{n}^{*}\right\rangle\left\langle e_{m}^{*}, e_{i} \circ e_{k}\right\rangle\right) .
\end{gathered}
$$

Similarly, the coefficients of $e_{m}^{*} \otimes e_{n}, e_{m} \otimes e_{n}^{*}$ and $e_{m}^{*} \otimes e_{n}^{*}$ on both sides of (7-5) are the same.

On the other hand, we prove that $S_{\alpha_{\mathscr{P} S \mathscr{I}}, \beta_{\mathscr{P} \mathcal{A} \mathscr{I}}}^{i}=0$ for $i=1,2,3,4$. We prove it

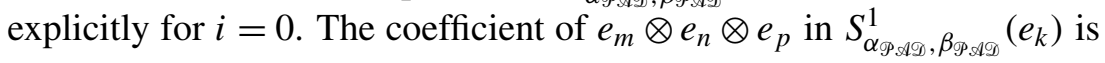

$$
\begin{gathered}
-\left\langle e_{j} \succ_{\bullet} e_{m}^{*}, e_{n}^{*}\right\rangle\left\langle e_{k} \bullet e_{j}^{*}, e_{p}^{*}\right\rangle+\left\langle e_{j} \bullet e_{m}^{*}, e_{n}^{*}\right\rangle\left\langle e_{k} \bullet e_{j}^{*}, e_{p}^{*}\right\rangle-\left\langle e_{j} \succ_{\bullet} e_{n}^{*}, e_{m}^{*}\right\rangle\left\langle e_{k} \bullet e_{j}^{*}, e_{p}^{*}\right\rangle \\
+\left\langle e_{j} \bullet e_{n}^{*}, e_{m}^{*}\right\rangle\left\langle e_{k} \bullet e_{j}^{*}, e_{p}^{*}\right\rangle-\left\langle\left(e_{k} \bullet e_{m}^{*}\right) \bullet e_{n}^{*}, e_{p}^{*}\right\rangle-\left\langle\left(e_{k} \bullet e_{n}^{*}\right) \bullet e_{m}^{*}, e_{p}^{*}\right\rangle \\
=\left\langle e_{j}, e_{m}^{*} \prec_{*} e_{n}^{*}\right\rangle\left\langle e_{k}, e_{j}^{*} \succ_{*} e_{p}^{*}\right\rangle+\left\langle e_{j}, e_{m}^{*} \succ_{*} e_{n}^{*}\right\rangle\left\langle e_{k}, e_{j}^{*} \succ_{*} e_{p}^{*}\right\rangle \\
+\left\langle e_{j}, e_{n}^{*} \prec_{*} e_{m}^{*}\right\rangle\left\langle e_{k}, e_{j}^{*} \succ_{*} e_{p}^{*}\right\rangle+\left\langle e_{j}, e_{n}^{*} \succ_{*} e_{m}^{*}\right\rangle\left\langle e_{k}, e_{j}^{*} \succ_{*} e_{p}^{*}\right\rangle \\
-\left\langle e_{k} \bullet\left(e_{m}^{*} * e_{n}^{*}+e_{n}^{*} * e_{m}^{*}\right), e_{p}^{*}\right\rangle \\
=\left\langle e_{k},\left(e_{m}^{*} * e_{n}^{*}+e_{n}^{*} * e_{m}^{*}\right) \succ_{\bullet} e_{p}^{*}-\left(e_{m}^{*} * e_{n}^{*}+e_{n}^{*} * e_{m}^{*}\right) \succ_{\bullet} e_{p}^{*}\right\rangle=0 .
\end{gathered}
$$

Similarly, the remaining coefficients, of $e_{m}^{*} \otimes e_{n} \otimes e_{p}, e_{m} \otimes e_{n}^{*} \otimes e_{p}, e_{m}^{*} \otimes e_{n}^{*} \otimes e_{p}$, $e_{m} \otimes e_{n} \otimes e_{p}^{*}, e_{m}^{*} \otimes e_{n} \otimes e_{p}^{*}, e_{m} \otimes e_{n}^{*} \otimes e_{p}^{*}$ and $e_{m}^{*} \otimes e_{n}^{*} \otimes e_{p}^{*}$, are all zero. A similar study shows that $S_{\alpha_{\mathscr{P} S \mathscr{I}}, \beta_{\mathscr{P} S \mathscr{D}}}^{1}\left(e_{k}^{*}\right)=0$. Hence $\mathscr{P} \mathscr{A} \mathscr{D}(A)$ is a prealternative bialgebra.

For $e_{i} \in A$, we have

$$
\begin{aligned}
\alpha_{\mathscr{P} \mathcal{A} \mathscr{D}}\left(e_{i}\right) & =\sum_{j} e_{j} \circ e_{i} \otimes e_{j}^{*}-e_{j} \otimes e_{i} \succ \bullet e_{j}^{*} \\
& =\sum_{j, m} e_{j} \circ e_{i} \otimes e_{j}^{*}-e_{j} \otimes e_{m}^{*}\left\langle e_{j}^{*}, e_{m} \circ e_{i}\right\rangle+e_{j} \otimes e_{m}\left\langle e_{i}, e_{j}^{*} \prec_{*} e_{m}^{*}\right\rangle \\
& =\sum_{j, m}\left\langle e_{i}, e_{j}^{*} \prec_{*} e_{m}^{*}\right\rangle e_{j} \otimes e_{m}=\alpha\left(e_{i}\right) .
\end{aligned}
$$


Similarly we have $\beta_{\mathscr{P} \mathscr{A}}\left(e_{i}\right)=\beta\left(e_{i}\right)$, so the inclusion $i_{1}: A \rightarrow A \oplus A^{*}$ is a homomorphism of prealternative bialgebras. Similarly, the inclusion $i_{2}: A^{*} \rightarrow A \oplus A^{*}$ is also a homomorphism of prealternative bialgebras.

Definition 7.9. Let $(A, \prec, \succ, \alpha, \beta)$ be a prealternative bialgebra. With the prealternative bialgebra structure given in Theorem 7.8, we call $A \oplus A^{*}$ a Drinfeld symplectic double of $A$ and denote it by $\mathscr{P} \mathscr{A D}(A)$.

Proposition 7.10. Let $(A, \prec, \succ, \alpha, \beta)$ be a prealternative bialgebra with $\alpha, \beta$ defined by (7-1), where $r_{\prec}=r_{\succ}=r \in A \otimes A$ and $r$ is a solution of the PA equations. Then $T_{r}$ is a homomorphism of prealternative bialgebras from the prealternative bialgebra given in Example 6.6 to $(A, \prec, \succ, \alpha, \beta)$.

Proof. Note that $(1 \otimes \alpha) r=r_{12} \prec r_{13}$ and $(\alpha \otimes 1) r=r_{13} \prec r_{23}$. Denote by $\prec_{*}$ and $\succ_{*}$ the prealternative algebras structure on $A^{*}$ induced by $\alpha^{*}$ and $\beta^{*}$, respectively, and define the prealternative algebra structure $\prec, \succ$ on $A$ by the linear maps $\gamma^{*}, \delta^{*}: A \otimes A \rightarrow A$, respectively. Then

$$
\begin{aligned}
T_{r}\left(a^{*} \prec_{*} b^{*}\right) & =\left\langle 1 \otimes\left(a^{*} \prec_{*} b^{*}\right), r\right\rangle=\left\langle 1 \otimes a^{*} \otimes b^{*},(1 \otimes \alpha) r\right\rangle \\
& =\left\langle 1 \otimes a^{*} \otimes b^{*}, r_{12} \prec r_{13}\right\rangle=T_{r}\left(a^{*}\right) \prec T_{r}\left(b^{*}\right),
\end{aligned}
$$

$\left(T_{r} \otimes T_{r}\right) \gamma\left(a^{*}\right)=\left\langle 1 \otimes 1 \otimes a^{*}, r_{13} \prec r_{23}\right\rangle=\left(1 \otimes 1 \otimes a^{*}\right)(\alpha \otimes 1) r=\alpha\left(T_{r}\left(a^{*}\right)\right)$, where $a^{*}, b^{*} \in A^{*}$. Similarly we have

$$
T_{r}\left(a^{*} \succ_{*} b^{*}\right)=T_{r}\left(a^{*}\right) \succ T_{r}\left(b^{*}\right) \quad \text { and } \quad\left(T_{r} \otimes T_{r}\right) \delta\left(a^{*}\right)=\beta\left(T_{r}\left(a^{*}\right)\right) .
$$

\section{PA equations and their properties}

The simplest way to satisfy the conditions of Theorem 7.7 is given as follows.

Proposition 8.1. Let $(A, \prec, \succ)$ be a prealternative algebra and $r \in A \otimes A$ be symmetric. Let $\alpha, \beta: A \rightarrow A \otimes A$ be two linear maps defined by (7-1). Then $(A, \prec, \succ, \alpha, \beta)$ is a prealternative bialgebra if $r$ satisfies PA-equations.

Proposition 8.2. Let $(A, \prec, \succ)$ be a prealternative algebra and $(\operatorname{Alt}(A), \circ)$ be the associated alternative algebra. Let $r \in A \otimes A$ be a symmetric solution of the PA equations in $A$. Then the prealternative algebra structure $\prec_{\bullet}, \succ_{\bullet}$ on the Drinfeld symplectic double $\mathscr{P} \mathscr{A D}(A)$ is given as

$$
\begin{aligned}
a^{*} \prec \bullet b^{*} & =a^{*} \prec_{*} b^{*}=\mathfrak{l}_{\circ}^{*}\left(T_{r}\left(b^{*}\right)\right) a^{*}-\mathfrak{r}_{\succ}^{*}\left(T_{r}\left(a^{*}\right)\right) b^{*}, \\
a^{*} \succ \bullet b^{*} & =a^{*} \succ_{*} b^{*}=\mathfrak{r}_{\circ}^{*}\left(T_{r}\left(a^{*}\right)\right) b^{*}-\mathfrak{r}_{\prec}^{*}\left(T_{r}\left(b^{*}\right)\right) a^{*}, \\
x \prec \bullet a^{*} & =x \prec T_{r}\left(a^{*}\right)+T_{r}\left(\mathfrak{r}_{\succ}^{*}(x) a^{*}\right)-\mathfrak{r}_{\succ}^{*}(x) a^{*}, \\
x \succ \bullet a^{*} & =\mathfrak{r}_{\circ}^{*}(x) a^{*}-T_{r}\left(\mathfrak{r}_{\circ}^{*}(x) a^{*}\right)+x \succ T_{r}\left(a^{*}\right), \\
a^{*} \prec \bullet x & =-T_{r}\left(\mathfrak{r}_{\circ}^{*}(x) a^{*}\right)+T_{r}\left(a^{*}\right) \prec x+\mathfrak{r}_{\circ}^{*}(x) a^{*},
\end{aligned}
$$




$$
a^{*} \succ \bullet x=T_{r}\left(a^{*}\right) \succ x+T_{r}\left(\mathfrak{l}_{<}^{*}(x) a^{*}\right)-\mathfrak{l}_{<}^{*}(x) a^{*},
$$

where $x \in A$ and $a^{*}, b^{*} \in A^{*}$, the prealternative algebra structure on $A^{*}$ is denoted by $\prec_{*}, \succ_{*}$, and the associated alternative algebra structure on $\operatorname{Alt}\left(A^{*}\right)$ is denoted by $*$.

Proof. Let $\left\{e_{1}, \ldots, e_{n}\right\}$ be a basis of $A$ and $\left\{e_{1}^{*}, \ldots, e_{n}^{*}\right\}$ be its dual. Suppose that

$$
e_{i} \prec e_{j}=\sum_{i, j} c_{i j}^{k} e_{k}, \quad e_{i} \succ e_{j}=\sum_{i, j} d_{i j}^{k} e_{k}, \quad r=\sum_{i, j} a_{i j} e_{i} \otimes e_{j}, \quad a_{i j}=a_{j i} .
$$

Then $T_{r}\left(e_{i}^{*}\right)=\sum_{k} a_{k i} e_{k}$. Thus for any $k, l$

$$
\begin{aligned}
e_{k}^{*} \prec_{*} e_{l}^{*} & =\sum_{s}\left\langle e_{k}^{*} \otimes e_{l}^{*}, \alpha\left(e_{s}\right)\right\rangle e_{s}^{*}=\sum_{i, s}\left(a_{i l}\left(c_{i s}^{k}+d_{i s}^{k}\right)-a_{k i} d_{s i}^{l}\right) e_{s}^{*} \\
& =\sum_{i, s}\left(a_{i l}\left\langle e_{i} \circ e_{s}, e_{k}^{*}\right\rangle-e_{k i}\left\langle e_{s} \succ e_{i}, e_{l}^{*}\right\rangle\right) e_{s}^{*}=\mathfrak{l}_{\circ}^{*}\left(T_{r}\left(e_{l}^{*}\right)\right) e_{k}^{*}-\mathfrak{r}_{\succ}^{*}\left(T_{r}\left(e_{k}^{*}\right)\right) e_{l}^{*} .
\end{aligned}
$$

So (8-1) holds. Similarly (8-2) holds. Therefore

$$
\begin{aligned}
\mathfrak{r}_{\succ_{*}}^{*}\left(e_{k}^{*}\right) e_{m} & =\sum_{s}\left\langle e_{m}, e_{k}^{*} \prec_{*} e_{s}^{*}\right\rangle e_{s}=\sum_{s}\left\langle e_{m}, \mathfrak{l}_{\circ}^{*}\left(T_{r}\left(e_{s}^{*}\right)\right) e_{k}^{*}-\mathfrak{r}_{\succ}^{*}\left(T_{r}\left(e_{k}^{*}\right)\right) e_{s}^{*}\right\rangle e_{s} \\
& =\sum_{s}\left\langle T_{r}\left(e_{s}^{*}\right) \circ e_{m}, e_{k}^{*}\right\rangle e_{s}-\left\langle e_{m} \succ T_{r}\left(e_{k}^{*}\right), e_{s}^{*}\right\rangle e_{s} \\
& =T_{r}\left(\mathfrak{r}_{\circ}^{*}\left(e_{m}\right) e_{k}^{*}\right)-e_{m} \succ T_{r}\left(e_{k}^{*}\right) .
\end{aligned}
$$

Hence (8-4) follows from the fact that $e_{m} \succ_{\bullet} e_{k}^{*}=\mathfrak{r}_{\circ}^{*}\left(e_{m}\right) e_{k}^{*}-\mathfrak{l}_{\prec_{*}}^{*}\left(e_{k}^{*}\right) e_{m}$. We can get the other equations similarly.

Proposition 8.3. Let $(A, \prec, \succ)$ be a prealternative algebra and $(\operatorname{Alt}(A), \circ)$ be the associated alternative algebra. Let $r \in A \otimes A$ be symmetric. Then $r$ is a solution of one of the $\mathrm{PA}_{j}^{i}$-equations for $i=1,2$ and $j=1,2,3$ if and only if $T_{r}$ satisfies

(8-7) $\quad T_{r}\left(a^{*}\right) \circ T_{r}\left(b^{*}\right)=T_{r}\left(\mathfrak{r}_{\prec}^{*}\left(T_{r}\left(a^{*}\right)\right) b^{*}+l_{\succ}^{*}\left(T_{r}\left(b^{*}\right)\right) a^{*}\right) \quad$ for all $a^{*}, b^{*} \in A^{*}$,

that is, $T_{r}$ is an $\mathbf{0}$-operator of $\operatorname{Alt}(A)$ associated to the bimodule $\left(A^{*}, \mathfrak{r}_{<}^{*}, l_{\succ}^{*}\right)$. So in this case the $\mathrm{PA}_{j}^{i}$ equations for $i=1,2$ and $j=1,2,3$ are all equivalent. Moreover, if $r$ is a solution of one of the $\mathrm{PA}_{j}^{i}$ equations for $i=1,2$ and $j=1,2,3$, then there is a prealternative algebra structure on $A^{*}$ given by

(8-8) $a^{*} \prec b^{*}=\mathfrak{l}_{\succ}^{*}\left(T_{r}\left(b^{*}\right)\right) a^{*} \quad$ and $\quad a^{*} \succ b^{*}=\mathfrak{r}_{<}^{*}\left(T_{r}\left(a^{*}\right)\right) b^{*} \quad$ for all $a^{*}, b^{*} \in A^{*}$.

The associated alternative algebra structure $\operatorname{Alt}\left(A^{*}\right)$ is the same as the one given by (8-1) and (8-2) that is induced by $r$ in the sense of coboundary prealternative bialgebras.

Proof. It is similar to the proof of Proposition 3.6. 
Definition 8.4. Let $(A, \prec, \succ)$ be a prealternative algebra. We call a bilinear form $\mathscr{B}: A \otimes A \rightarrow \mathbf{k}$ a 2-cocycle of $A$ if

$$
\mathscr{B}(x \circ y, z)=\mathscr{B}(x, y \succ z)+\mathscr{B}(y, z \prec x) \quad \text { for all } x, y, z \in A .
$$

Proposition 8.5. Let $(A, \prec, \succ)$ be a prealternative algebra and $(\operatorname{Alt}(A), \circ)$ be the associated alternative algebra. Let $\mathscr{B}$ be a 2-cocycle of $(A, \prec, \succ)$. Then the bilinear form $\omega$ defined by

$$
\omega(x, y)=\mathscr{B}(x, y)-\mathscr{B}(y, x) \text { for all } x, y \in A
$$

is a closed form on $\operatorname{Alt}(A)$.

Proof. Straightforward.

Proposition 8.6. Let $(A, \prec, \succ)$ be a prealternative algebra and let $r \in A \otimes A$. Suppose $r$ is symmetric and nondegenerate. Then $r$ is a solution of one of the $\mathrm{PA}_{j}^{i}$ equations for $i=1,2$ and $j=1,2,3$ in $(A, \prec, \succ)$ if and only if the (nondegenerate) bilinear form $\mathscr{B}$ induced by $r$ through (1-8) is a 2-cocycle of $(A, \prec, \succ)$.

Proof. Let $r=\sum_{i} a_{i} \otimes b_{i}$. Since $r$ is symmetric, we have $\sum_{i} a_{i} \otimes b_{i}=\sum_{i} b_{i} \otimes a_{i}$. Therefore $T_{r}\left(v^{*}\right)=\sum_{i}\left\langle v^{*}, a_{i}\right\rangle b_{i}=\sum_{i}\left\langle v^{*}, b_{i}\right\rangle a_{i}$ for any $v^{*} \in A^{*}$. Since $r$ is nondegenerate, for any $x, y, z \in A$ there exist $u^{*}, v^{*}, w^{*} \in A^{*}$ such that $x=T_{r}\left(u^{*}\right)$, $y=T_{r}\left(v^{*}\right)$ and $z=T_{r}\left(w^{*}\right)$. Therefore

$$
\begin{aligned}
\mathscr{B}(x, z \circ y) & =\left\langle u^{*}, T_{r}\left(w^{*}\right) \circ T_{r}\left(v^{*}\right)\right\rangle \\
& =\sum_{i, j}\left\langle w^{*}, b_{i}\right\rangle\left\langle v^{*}, b_{j}\right\rangle\left\langle u^{*}, a_{i} \circ a_{j}\right\rangle=\left\langle u^{*} \otimes v^{*} \otimes w^{*}, r_{13} \circ r_{12}\right\rangle, \\
\mathscr{B}(y, x \prec z\rangle & =\left\langle v^{*}, T_{r}\left(u^{*}\right) \prec T_{r}\left(w^{*}\right)\right\rangle \\
& =\sum_{i, j}\left\langle u^{*}, b_{i}\right\rangle\left\langle w^{*}, b_{j}\right\rangle\left\langle v^{*}, a_{i} \prec a_{j}\right\rangle=\left\langle u^{*} \otimes v^{*} \otimes w^{*}, r_{12} \prec r_{23}\right\rangle, \\
\mathscr{B}(y \succ x, z) & =\left\langle T_{r}\left(v^{*}\right) \succ T_{r}\left(u^{*}\right), w^{*}\right\rangle \\
& =\sum_{i, j}\left\langle v^{*}, b_{i}\right\rangle\left\langle u^{*}, b_{j}\right\rangle\left\langle w^{*}, a_{i} \succ a_{j}\right\rangle=\left\langle u^{*} \otimes v^{*} \otimes w^{*}, r_{23} \succ r_{13}\right\rangle .
\end{aligned}
$$

Hence $\mathscr{B}$ is a 2-cocycle of $(A, \prec, \succ)$ if and only if the second of (7-3) holds. By Proposition 8.3, the conclusion follows.

Corollary 8.7. Let $(A, \prec, \succ)$ be a prealternative algebra and $r \in A \otimes A$. Assume $r$ is symmetric and there exists a nondegenerate symmetric bilinear form $h(x, y)$ on A that is associative in that

$$
h(x \prec y, z)=h(x, y \succ z) \quad \text { for all } x, y, z \in A \text {. }
$$


Define a linear map $\varphi: A \rightarrow A^{*}$ by $\langle\varphi(x), y\rangle=h(x, y)$. Then $\tilde{T}_{r}=T_{r} \varphi: A \rightarrow A$ is an $\mathbf{O}$-operator associated to the bimodule $\left(A, \mathfrak{l}_{\succ}, \mathfrak{r}_{<}\right)$if and only if $r$ is a symmetric solution of the PA equations. In this case, $\tilde{T}_{r}$ satisfies the equation

$$
\tilde{T}_{r}(x) \circ \tilde{T}_{r}(y)=\tilde{T}_{r}\left(\tilde{T}_{r}(x) \succ y+x \prec \tilde{T}_{r}(y)\right) .
$$

So we can define a prealternative algebra structure on A by

$$
\begin{aligned}
& x \prec y=x \prec \tilde{T}_{r}(y), \\
& x \succ y=\tilde{T}_{r}(x) \succ y .
\end{aligned}
$$

Proof. It follows from a proof similar to that of Corollary 3.7.

Remark 8.8. A symmetric bilinear form on a prealternative algebra $(A, \prec, \succ)$ satisfying $(8-10)$ is a 2-cocycle of $(A, \prec, \succ)$.

By a proof similar to that of Proposition 3.9, we have this:

Proposition 8.9. Let $(A, \circ)$ be an alternative algebra. Let $(V, L, R)$ be a bimodule of $A$ and $\left(V^{*}, R^{*}, L^{*}\right)$ be the dual bimodule. Suppose that $T: V \rightarrow A$ is an O-operator associated to $(V, L, R)$. Then $r=T+\sigma(T)$ is a symmetric solution of the PA equations in $T(V) \ltimes_{0, L^{*}, R^{*}, 0} V^{*}$, where $T(V) \subset A$ is a prealternative algebra given by (2-9) and $\left(V^{*}, 0, L^{*}, R^{*}, 0\right)$ is a bimodule of $T(V)$.

Corollary 8.10. Let $(A, \prec, \succ)$ be a prealternative algebra. Then

$$
r=\sum_{i}\left(e_{i} \otimes e_{i}^{*}+e_{i}^{*} \otimes e_{i}\right)
$$

is a symmetric solution of the PA equations in $A \ltimes_{0, L_{\gtrless}^{*}, r_{*}^{*}, 0} A^{*}$, where $\left\{e_{1}, \ldots, e_{n}\right\}$ is a basis of $A$ and $\left\{e_{1}^{*}, \ldots, e_{n}^{*}\right\}$ is its dual. Moreover, $r$ is nondegenerate and the induced 2-cocycle $\mathscr{B}$ of $A \ltimes_{0, r_{*}^{*}, r^{*}, 0} A^{*}$ is given by (3-2).

Proof. Use Proposition 8.9 with $V=A,(L, R)=\left(\mathfrak{l}_{\succ}, \mathfrak{r}_{<}\right)$and $T=\mathrm{id}$.

Corollary 8.11. Let $(A, \prec, \succ)$ be a prealternative algebra and $(\operatorname{Alt}(A), \circ)$ be the associated alternative algebra. If $r$ is a nondegenerate symmetric solution of the $P A$ equations in $A$, then there is a new compatible prealternative algebra structure on $\operatorname{Alt}(A)$ given by

$$
\begin{aligned}
& x \prec^{\prime} y=T_{r}\left(l_{\succ}^{*}(y) T_{r}^{-1}(x)\right), \\
& x \succ^{\prime} y=T_{r}\left(\mathfrak{r}_{\prec}^{*}(x) T_{r}^{-1}(y)\right) \quad \text { for all } x, y \in A,
\end{aligned}
$$

which is just the prealternative algebra structure given by

$$
\begin{aligned}
& \mathscr{B}\left(x \prec^{\prime} y, z\right)=\mathscr{B}(x, y \succ z), \\
& \mathscr{B}\left(x \succ^{\prime} y, z\right)=\mathscr{B}(y, z \prec x) \quad \text { for all } x, y, z \in A,
\end{aligned}
$$

where $\mathscr{B}$ is the nondegenerate symmetric 2-cocycle of $A$ induced by $r$ through (1-8). 
Proposition 8.12. Let $(A, \prec, \succ, \alpha, \beta)$ be a prealternative bialgebra arising from a symmetric solution $r$ of the PA equations and let the corresponding matched pair of prealternative algebras be $\left(A^{*},-\mathfrak{r}_{\succ}^{*}, \mathfrak{l}_{\circ}^{*}, \mathfrak{r}_{\circ}^{*},-\mathfrak{l}_{<}^{*},-\mathfrak{r}_{\succ_{*}^{*}}^{*}, \mathfrak{l}_{*}^{*}, \mathfrak{r}_{*}^{*},-\mathfrak{l}_{<_{*}^{*}}^{*}\right)$.

(1) As prealternative algebras,

$$
A \bowtie_{-\mathfrak{r}_{\succ}^{*}, \mathfrak{r}_{0}^{*}, \mathfrak{r}_{0}^{*},-\mathfrak{l}_{<}^{*}}^{-\mathfrak{r}_{<}^{*}, \mathfrak{r}^{*},-\mathfrak{r}^{*}} A^{*} \quad \text { and } \quad A \ltimes_{-\mathfrak{r}_{\succ}^{*},,_{0}^{*}, \mathfrak{r}_{\circ}^{*},-\mathfrak{l}_{<}^{*}} A^{*}
$$

are isomorphic.

(2) The symmetric solutions of the PA equations are in one-to-one correspondence with linear maps $T_{r}: A^{*} \rightarrow A$ whose graphs are Lagrangian prealternative subalgebras (with respect to the bilinear form (3-10) of $A \ltimes_{-\mathfrak{r}_{>}^{*},,_{0}^{*}, r_{\circ}^{*},-\mathfrak{l}_{<}^{*}} A^{*}$.

Proof. It is similar to the proof of Proposition 3.13.

\section{Comparison between alternative D-bialgebras and prealternative bialgebras}

The results in the previous sections allow us to compare alternative D-bialgebras (see the appendix) and prealternative bialgebras in terms of matched pairs of alternative algebras; alternative algebra structures on the direct sum of the alternative algebras in the matched pairs; bilinear forms on the direct sum of the alternative algebras in the matched pairs; double structures on the direct sum of the alternative algebras in the matched pairs; algebraic equations associated to coboundary cases, nondegenerate solutions; 0 -operators of alternative algebras; and constructions from prealternative algebras. See Table 1.

From the table, we observe that there is a clear analogy between alternative D-bialgebras and prealternative bialgebras. Due to the correspondences between certain symmetries and skew-symmetries appearing in the table, we regard it as a kind of duality.

\section{Appendix: Another approach to alternative D-bialgebras}

In this section we prove the main results of [Goncharov 2007] by using a slightly different method (in fact, we will prove some results that are a little stronger than those there). There will be some results that were not presented there, such as the Drinfeld double theorem for an alternative D-bialgebra (Theorem A.10) and a homomorphism property of an alternative D-bialgebra (Theorem A.11). We omit the proofs since they are quite similar to the study of prealternative bialgebras.

Theorem A.1. Let $(A, \circ)$ be an alternative algebra and $\left(A^{*}, *\right)$ be an alternative algebra induced by a linear map $\Delta: A \rightarrow A \otimes A$. Then $(A, \circ, \Delta)$ is an alternative $D$-bialgebra if and only if $\left(A, A^{*}, \mathfrak{r}_{\circ}^{*}, \mathfrak{l}_{0}^{*}, \mathfrak{r}_{*}^{*}, \mathfrak{l}_{*}^{*}\right)$ is a matched pair of alternative algebras. 
Algebras

Alternative D-bialgebras Prealternative bialgebras

Matched pairs of alternative

$\left(A, A^{*}, \mathfrak{r}_{\circ}^{*}, \mathfrak{l}_{\circ}^{*}, \mathfrak{r}_{*}^{*}, \mathfrak{l}_{*}^{*}\right)$

$\left(\operatorname{Alt}(A), \operatorname{Alt}\left(A^{*}\right), \mathfrak{r}_{\prec}^{*}, l_{\succ}^{*}\right.$,

algebras

$\left.\mathfrak{r}_{\prec_{*}^{*}}^{*}, \mathfrak{l}_{\succ_{*}^{*}}^{*}\right)$

Alternative algebra

alternative

phase spaces

structures on the direct sum

analogues of Manin

of the alternative algebras

triples

in the matched pairs

Bilinear forms on

the direct sum of the

alternative algebras

in the matched pairs

\begin{tabular}{cc} 
symmetric & skew-symmetric \\
\hline $\begin{array}{c}\left\langle x+a^{*}, y+b^{*}\right\rangle \\
=\left\langle x, b^{*}\right\rangle+\left\langle a^{*}, y\right\rangle\end{array}$ & $\begin{array}{c}\left.x+a^{*}, y+b^{*}\right\rangle \\
=-\left\langle x, b^{*}\right\rangle+\left\langle a^{*}, y\right\rangle\end{array}$ \\
\hline invariant & symplectic forms
\end{tabular}

Double structures on

Drinfeld's doubles

Drinfeld's symplectic

the direct sum of the doubles

alternative algebras

in the matched pairs

Algebraic equations associated to

coboundary cases

skew-symmetric solutions

symmetric solutions

alternative YBEs in

PA-equations in alternative algebras prealternative algebras

Nondegenerate solutions

symplectic forms of

2-cocycles of prealternative alternative algebras algebras

O-operators associated to $\left(\mathfrak{r}_{\circ}^{*}, \mathfrak{l}_{\circ}^{*}\right)$ associated to $\left(\mathfrak{r}_{\prec}^{*}, \mathfrak{l}_{\succ}^{*}\right)$

skew-symmetric parts

symmetric parts

Constructions from prealternative algebras

$$
\begin{array}{cc}
r= & r= \\
\sum_{i=1}^{n}\left(e_{i} \otimes e_{i}^{*}-e_{i}^{*} \otimes e_{i}\right) & \sum_{i=1}^{n}\left(e_{i} \otimes e_{i}^{*}+e_{i}^{*} \otimes e_{i}\right) \\
\hline \text { induced bilinear forms } & \text { induced bilinear forms } \\
\left\langle x+a^{*}, y+b^{*}\right\rangle & \left\langle x+a^{*}, y+b^{*}\right\rangle \\
=-\left\langle x, b^{*}\right\rangle+\left\langle a^{*}, y\right\rangle & =\left\langle x, b^{*}\right\rangle+\left\langle a^{*}, y\right\rangle
\end{array}
$$

Table 1. Comparison between alternative D-bialgebras and prealternative bialgebras

Theorem A.2. Let $(A, \circ, \Delta)$ be an alternative algebra equipped with a linear map $\Delta: A \rightarrow A \otimes A$ such that $\Delta^{*}: A^{*} \otimes A^{*} \rightarrow A^{*}$ induces an alternative algebra structure on $A^{*}$. Then $\left(A, A^{*}, \mathfrak{r}_{\circ}^{*}, \mathfrak{l}_{\circ}^{*}, \mathfrak{r}_{*}^{*}, \mathfrak{r}_{*}^{*}\right)$ is a matched pair of alternative algebras if and 
only if the following equations hold:

$$
\begin{aligned}
& \Delta(x \circ y)=\left(-\mathfrak{l}_{\circ}(x) \otimes 1+1 \otimes \operatorname{Ass}_{\circ}(x)\right) \Delta(y)+\left(\mathfrak{r}_{\circ}(y) \otimes 1\right) \Delta(x) \\
& +\left(\mathfrak{r}_{\circ}(y) \otimes 1-1 \otimes \mathfrak{l}_{\circ}(y)\right) \sigma \Delta(x), \\
& \Delta(x \circ y)=\left(\operatorname{Ass}_{\circ}(y) \otimes 1-1 \otimes \mathfrak{r}_{\circ}(y)\right) \Delta(x)+\left(1 \otimes \mathfrak{l}_{\circ}(x)\right) \Delta(y) \\
& +\left(1 \otimes \mathfrak{l}_{\circ}(x)-\mathfrak{r}_{\circ}(x) \otimes 1\right) \sigma \Delta(y),
\end{aligned}
$$

(A-3) $\Delta(x \circ y+y \circ x)=\left(\mathfrak{r}_{\circ}(y) \otimes 1+1 \otimes \mathfrak{l}_{\circ}(y)\right) \Delta(x)$

$$
+\left(1 \otimes \mathfrak{l}_{\circ}(x)+\mathfrak{r}_{\circ}(x) \otimes 1\right) \Delta(y),
$$

$(\mathrm{A}-4) \quad(\Delta+\sigma \Delta)(x \circ y)=\left(\mathfrak{r}_{\circ}(y) \otimes 1\right) \Delta(x)+\left(1 \otimes \mathfrak{r}_{\circ}(y)\right) \sigma \Delta(x)$

$$
+\left(\mathfrak{l}_{\circ}(x) \otimes 1\right) \sigma \Delta(y)+\left(1 \otimes \mathfrak{l}_{\circ}(x)\right) \Delta(y),
$$

where $x, y \in A$, the multiplication $*$ is induced by $\Delta$, and $\mathrm{Ass}_{\circ}=\mathfrak{l}_{\circ}+\mathfrak{r}_{\circ}$.

Remark A.3. Equations (A-1) and (A-4) have already appeared as [Goncharov 2007, Lemma 2].

Definition A.4. An alternative D-bialgebra $(A, \circ, \Delta)$ is called coboundary if there exists an $r \in A \otimes A$ such that $\Delta$ is given by

$$
\Delta(x)=\left(\mathfrak{r}_{\circ}(x) \otimes 1-1 \otimes \mathfrak{l}_{\circ}(x)\right) r \quad \text { for all } x \in A .
$$

This definition is the same as Definition 3.3.

Lemma A.5. Let $A$ be a vector space. Then a linear map $\Delta: A \rightarrow A \otimes A$ induces an alternative algebra structure on $A^{*}$ if and only if for any $x, y \in A$

$(\Delta \otimes 1) \Delta(x)+(\sigma \otimes 1)(\Delta \otimes 1) \Delta(x)=(1 \otimes \Delta) \Delta(x)+(\sigma \otimes 1)(1 \otimes \Delta) \Delta(x)$, $(\Delta \otimes 1) \Delta(x)+(1 \otimes \sigma)(\Delta \otimes 1) \Delta(x)=(1 \otimes \Delta) \Delta(x)+(1 \otimes \sigma)(1 \otimes \Delta) \Delta(x)$.

Definition A.6. Let $(A, \circ)$ be an alternative algebra. The following equations are called the $A_{i}$-equations in $A$ for $i=1,2$ :

$$
\begin{aligned}
& A_{1}=r_{23} \circ r_{12}-r_{13} \circ r_{23}-r_{12} \circ r_{13}=0, \\
& A_{2}=r_{12} \circ r_{23}-r_{23} \circ r_{13}-r_{13} \circ r_{12}=0 .
\end{aligned}
$$

Note that $A_{1}$ given by (A-6) is exactly $C_{A}(r)$ given by (3-5).

Proposition A.7. Let $(A, \circ)$ be an alternative algebra and $r \in A \otimes A$. If $r$ is skew-symmetric, then $A_{1}$ and $A_{2}$ are the same.

Proposition A.8. Let $(A, \circ)$ be an alternative algebra. Let $r \in A \otimes A$ be skewsymmetric. Define a linear map $\Delta: A \rightarrow A \otimes A$ by (A-5). Then $\Delta$ induces an alternative algebra structure on $A^{*}$ if and only if for any $x \in A$

$-\left(\mathfrak{r}_{\circ}(x) \otimes 1 \otimes 1\right) A_{1}-\left(1 \otimes \mathfrak{r}_{\circ}(x) \otimes 1\right) A_{2}+\left(1 \otimes 1 \otimes \mathfrak{l}_{\circ}(x)\right)\left(A_{1}+A_{2}\right)=0$,

$-\left(\mathfrak{r}_{\circ}(x) \otimes 1 \otimes 1\right)\left(A_{1}+A_{2}\right)+\left(1 \otimes \mathfrak{l}_{\circ}(x) \otimes 1\right) A_{2}+\left(1 \otimes 1 \otimes \mathfrak{l}_{\circ}(x)\right) A_{1}=0$, 
Theorem A.9 [Goncharov 2007, Theorem 2]. Suppose $(A, \circ)$ is an alternative algebra and $r \in A \otimes A$. Let $\Delta: A \rightarrow A$ be a linear map defined by (A-5). If $r$ is a skew-symmetric solution of the alternative Yang-Baxter equation in A, then $(A, \circ, \Delta)$ is an alternative D-bialgebra.

Theorem A.10. Let $\left(A, \circ, \Delta_{A}\right)$ be an alternative D-bialgebra. Then there is a canonical alternative bialgebra structure on $A \oplus A^{*}$ such that the inclusion $i_{1}$ : $A \rightarrow A \oplus A^{*}$ is a homomorphism of alternative D-bialgebras, where the alternative bialgebra structure on $A$ is given by $\left(A, \circ,-\Delta_{A}\right)$ and the inclusion $i_{2}$ of $A^{*}$ into $A \oplus A^{*}$ is a homomorphism of alternative D-bialgebras, where the alternative bialgebra structure on $A^{*}$ is given by $\left(A^{*}, *, \Delta_{B}\right)$, where $*$ is induced by $\Delta_{A}$, and where the alternative algebra structure $\circ$ on $A$ is induced by $\Delta_{B}: A^{*} \rightarrow A^{*} \otimes A^{*}$.

Theorem A.11. Let $(A, \circ, \Delta)$ be an alternative D-bialgebra arising from a solution $r$ of the alternative Yang-Baxter equation in A. Then $T_{r}$ is a homomorphism of alternative D-bialgebras from $\left(A^{*}, *, \delta\right)$ to $(A, \circ,-\Delta)$, where $*$ is induced by $\Delta$ and the alternative algebra structure $\circ$ on $A$ is induced by $\delta: A^{*} \rightarrow A^{*} \otimes A^{*}$.

\section{Acknowledgements}

We thank Professor J.-L. Loday and the referees for important suggestions. Ni thanks Professor V.N. Zhelyabin for kindly sending his paper to him.

\section{References}

[Aguiar and Sottile 2005] M. Aguiar and F. Sottile, "Cocommutative Hopf algebras of permutations and trees", J. Algebraic Combin. 22:4 (2005), 451-470. MR 2006g:16080 Zbl 1094.16024

[Aguiar and Sottile 2006] M. Aguiar and F. Sottile, "Structure of the Loday-Ronco Hopf algebra of trees”, J. Algebra 295:2 (2006), 473-511. MR 2006k:16078 Zbl 1099.16015

[Bai 2006] C. Bai, "A further study on non-abelian phase spaces: Left-symmetric algebraic approach and related geometry", Rev. Math. Phys. 18:5 (2006), 545-564. MR 2007f:17044 Zbl 1110.17008

[Bai 2007] C. Bai, "A unified algebraic approach to the classical Yang-Baxter equation", J. Phys. A 40:36 (2007), 11073-11082. MR 2009b:17014 Zbl 1118.17008

[Baxter 1960] G. Baxter, "An analytic problem whose solution follows from a simple algebraic identity”, Pacific J. Math. 10 (1960), 731-742. MR 22 \#9990 Zbl 0095.12705

[Burde 2006] D. Burde, "Left-symmetric algebras, or pre-Lie algebras in geometry and physics", Cent. Eur. J. Math. 4:3 (2006), 323-357. MR 2007c:17001 Zbl 1151.17301

[Chapoton 2002] F. Chapoton, "Un théorème de Cartier-Milnor-Moore-Quillen pour les bigèbres dendriformes et les algèbres braces", J. Pure Appl. Algebra 168:1 (2002), 1-18. MR 2002k:18013 Zbl 0994.18006

[Chari and Pressley 1994] V. Chari and A. Pressley, A guide to quantum groups, Cambridge University Press, 1994. MR 95j:17010 Zbl 0839.17009

[Drinfeld 1983] V. G. Drinfel'd, "Hamiltonian structures on Lie groups, Lie bialgebras and the geometric meaning of classical Yang-Baxter equations", Dokl. Akad. Nauk SSSR 268:2 (1983), 285287. In Russian; translated in Soviet Math. Dokl. 27 (1983), 68-71. MR 84i:58044 Zbl 0526.58017 
[Ebrahimi-Fard and Guo 2007] K. Ebrahimi-Fard and L. Guo, "Rota-Baxter algebras in renormalization of perturbative quantum field theory", pp. 47-105 in Universality and renormalization, edited by I. Binder and D. Kreimer, Fields Inst. Commun. 50, Amer. Math. Soc., Providence, RI, 2007. MR 2008i:81108 Zbl 1148.81018

[Frabetti 1997] A. Frabetti, "Dialgebra homology of associative algebras", C. R. Acad. Sci. Paris Sér. I Math. 325:2 (1997), 135-140. MR 98f:16010 Zbl 0883.16005

[Frabetti 1998] A. Frabetti, "Leibniz homology of dialgebras of matrices", J. Pure Appl. Algebra 129:2 (1998), 123-141. MR 99g:16013 Zbl 0936.17003

[Goncharov 2007] M. E. Goncharov, "The classical Yang-Baxter equation on alternative algebras: The alternative $D$-bialgebra structure on the Cayley-Dickson matrix algebra", Sibirsk. Mat. Zh. 48:5 (2007), 1008-1024. In Russian; translated in Siberian Math. J. 48:5 (2007), 809-823. MR 2009i:17047 Zbl 1164.17022

[Holtkamp 2004] R. Holtkamp, “On Hopf algebra structure over operads”, preprint, version 2, 2004. arXiv math.RA/0407074v2

[Jacobson 1968] N. Jacobson, Structure and representations of Jordan algebras, Amer. Math. Soc. Colloq. Publ. 39, American Mathematical Society, Providence, R.I., 1968. MR 40 \#4330 Zbl 0218. 17010

[Kupershmidt 1994] B. A. Kupershmidt, "Non-abelian phase spaces”, J. Phys. A 27:8 (1994), 28012809. MR 95g:17031 Zbl 0842.58030

[Kupershmidt 1999] B. A. Kupershmidt, "What a classical $r$-matrix really is", J. Nonlinear Math. Phys. 6:4 (1999), 448-488. MR 2001a:17023 Zbl 1015.17015

[Kuz'min and Shestakov 1995] E. N. Kuz'min and I. P. Shestakov, “Non-associative structures”, pp. 197-280 in Algebra, VI, Encyclopaedia Math. Sci. 57, Springer, Berlin, 1995. MR 91i:17001

[Loday 2001] J.-L. Loday, “Dialgebras”, pp. 7-66 in Dialgebras and related operads, Lecture Notes in Math. 1763, Springer, Berlin, 2001. MR 2002i:17004 Zbl 0999.17002

[Loday 2002] J.-L. Loday, “Arithmetree”, J. Algebra 258:1 (2002), 275-309. MR 2004c:05053 Zbl 1063.16044

[Loday 2004] J.-L. Loday, "Scindement d'associativité et algèbres de Hopf”, pp. 155-172 in Actes des Journées Mathématiques à la Mémoire de Jean Leray, Sémin. Congr. 9, Soc. Math. France, Paris, 2004. MR 2006a:16054 Zbl 1073.16032

[Loday 2008] J.-L. Loday, Generalized bialgebras and triples of operads, Astérisque 320, Société Mathématique de France, Paris, 2008. MR 2010f:18007 Zbl 1178.18001

[Ronco 2002] M. Ronco, "Eulerian idempotents and Milnor-Moore theorem for certain non-cocommutative Hopf algebras", J. Algebra 254:1 (2002), 152-172. MR 2003f:16064 Zbl 1017.16033

[Rota 1969] G.-C. Rota, "Baxter algebras and combinatorial identities, I", Bull. Amer. Math. Soc. 75:2 (1969), 330-334. MR 39 \#5387 Zbl 0192.33801

[Schafer 1952] R. D. Schafer, "Representations of alternative algebras", Trans. Amer. Math. Soc. 72 (1952), 1-17. MR 13,527b Zbl 0046.03503

[Schafer 1954] R. D. Schafer, "On the algebras formed by the Cayley-Dickson process", Amer. J. Math. 76 (1954), 435-446. MR 15,774d Zbl 0059.02901

[Schafer 1995] R. D. Schafer, An introduction to nonassociative algebras, Dover, New York, 1995. MR 96j:17001 Zbl 0145.25601

[Zhelyabin 1997] V. N. Zhelyabin, "Jordan bialgebras and their connection with Lie bialgebras", Algebra i Logika 36:1 (1997), 3-25, 117. In Russian; translated in Algebra and Logic 36 (1997), 1-15. MR 98d:17036 Zbl 0935.17014 
Received August 14, 2009. Revised July 8, 2010.

XIANG Ni

Chern Institute of Mathematics \& LPMC NANKAI UNIVERSITY

TIANJIN 300071

CHINA

xiangn_math@yahoo.cn

Chengming Bai

Chern Institute of Mathematics \& LPMC NANKAI UNIVERSITY

TIANJIN 300071

CHINA

baicm@nankai.edu.cn 


\title{
PACIFIC JOURNAL OF MATHEMATICS
}

\author{
http://www.pjmath.org \\ Founded in 1951 by \\ E. F. Beckenbach (1906-1982) and F. Wolf (1904-1989)
}

\section{EDITORS}

V. S. Varadarajan (Managing Editor)

Department of Mathematics

University of California

Los Angeles, CA 90095-1555

pacific@math.ucla.edu

Vyjayanthi Chari

Department of Mathematics

University of California

Riverside, CA 92521-0135

chari@math.ucr.edu

Robert Finn

Department of Mathematics Stanford University

Stanford, CA 94305-2125

finn@math.stanford.edu

Kefeng Liu

Department of Mathematics

University of California

Los Angeles, CA 90095-1555

liu@math.ucla.edu
Darren Long

Department of Mathematics

University of California

Santa Barbara, CA 93106-3080

long@math.ucsb.edu

Jiang-Hua Lu

Department of Mathematics

The University of Hong Kong

Pokfulam Rd., Hong Kong jhlu@maths.hku.hk

Alexander Merkurjev

Department of Mathematics

University of California

Los Angeles, CA 90095-1555

merkurev@math.ucla.edu
Sorin Popa

Department of Mathematics University of California

Los Angeles, CA 90095-1555 popa@math.ucla.edu

Jie Qing

Department of Mathematics

University of California

Santa Cruz, CA 95064

qing@cats.ucsc.edu

Jonathan Rogawski

Department of Mathematics

University of California

Los Angeles, CA 90095-1555

jonr@math.ucla.edu

\section{PRODUCTION}

pacific@math.berkeley.edu

\begin{abstract}
Silvio Levy, Scientific Editor Matthew Cargo, Senior Production Editor
\end{abstract}
ACADEMIA SINICA, TAIPEI

CALIFORNIA INST. OF TECHNOLOGY

INST. DE MATEMÁTICA PURA E APLICADA

KEIO UNIVERSITY

MATH. SCIENCES RESEARCH INSTITUTE

NEW MEXICO STATE UNIV.

OREGON STATE UNIV.

\section{SUPPORTING INSTITUTIONS}

STANFORD UNIVERSITY
UNIV. OF BRITISH COLUMBIA
UNIV. OF CALIFORNIA, BERKELEY
UNIV. OF CALIFORNIA, DAVIS
UNIV. OF CALIFORNIA, LOS ANGELES
UNIV. OF CALIFORNIA, RIVERSIDE
UNIV. OF CALIFORNIA, SAN DIEGO
UNIV. OF CALIF., SANTA BARBARA

UNIV. OF CALIF., SANTA CRUZ

UNIV. OF MONTANA

UNIV. OF OREGON

UNIV. OF SOUTHERN CALIFORNIA

UNIV. OF UTAH

UNIV. OF WASHINGTON

WASHINGTON STATE UNIVERSITY

These supporting institutions contribute to the cost of publication of this Journal, but they are not owners or publishers and have no responsibility for its contents or policies.

See inside back cover or www.pjmath.org for submission instructions.

The subscription price for 2010 is US \$420/year for the electronic version, and \$485/year for print and electronic.

Subscriptions, requests for back issues from the last three years and changes of subscribers address should be sent to Pacific Journal of Mathematics, P.O. Box 4163, Berkeley, CA 94704-0163, U.S.A. Prior back issues are obtainable from Periodicals Service Company, 11 Main Street, Germantown, NY 12526-5635. The Pacific Journal of Mathematics is indexed by Mathematical Reviews, Zentralblatt MATH, PASCAL CNRS Index, Referativnyi Zhurnal, Current Mathematical Publications and the Science Citation Index.

The Pacific Journal of Mathematics (ISSN 0030-8730) at the University of California, c/o Department of Mathematics, 969 Evans Hall, Berkeley, CA 94720-3840, is published monthly except July and August. Periodical rate postage paid at Berkeley, CA 94704, and additional mailing offices. POSTMASTER: send address changes to Pacific Journal of Mathematics, P.O. Box 4163, Berkeley, CA 94704-0163.

PJM peer review and production are managed by EditFLOW ${ }^{\mathrm{TM}}$ from Mathematical Sciences Publishers.

PUBLISHED BY PACIFIC JOURNAL OF MATHEMATICS

at the University of California, Berkeley 94720-3840

A NON-PROFIT CORPORATION

Typeset in LATEX

Copyright $(\mathrm{C} 2010$ by Pacific Journal of Mathematics 


\section{PACIFIC JOURNAL OF MATHEMATICS}

Volume $248 \quad$ No. $2 \quad$ December 2010

Topological description of Riemannian foliations with dense leaves

Jesús A. ÁlVAREZ LóPEZ and Alberto CANDEL

The nonexistence of quasi-Einstein metrics

JEFFREY S. CASE

Twisted symmetric group actions

AKINARI HoSHI and MING-CHANG KANG

Optimal transportation and monotonic quantities on evolving manifolds

305

HONG HUANG

Hopf structures on the Hopf quiver $Q(\langle g\rangle, g)$

HuA-Lin HuANG, YU Ye and QING ZHaO

Minimal surfaces in $S^{3}$ foliated by circles

Nikolai KuteV and VelichKa Milousheva

Prealternative algebras and prealternative bialgebras

XIANG Ni and CHENGMING BAI

Some remarks about closed convex curves

Ke OU and Shengliang PAN

Orbit correspondences for real reductive dual pairs

SHU-YEN PAN

Graphs of bounded degree and the $p$-harmonic boundary

Michael J. Puls

Invariance of the BFV complex

FLORIAN SCHÄTZ

Some elliptic PDEs on Riemannian manifolds with boundary

YANNICK SIRE and ENRICO VALDINOCI

Representations of Lie superalgebras in prime characteristic, III 